\title{
Spherical-harmonic tensors
}

\author{
Francisco Gonzalez Ledesma ${ }^{1}$ and Matthew Mewes $\odot^{2}$ \\ ${ }^{1}$ Department of Physics, Florida State University, Tallahassee, Florida 32306, USA \\ ${ }^{2}$ Physics Department, California Polytechnic State University, San Luis Obispo, California 93407, USA
}

(Received 19 May 2020; accepted 28 September 2020; published 12 October 2020)

\begin{abstract}
The connection between spherical harmonics and symmetric tensors is explored. For each spherical harmonic, a corresponding traceless symmetric tensor is constructed. These tensors are then extended to include nonzero traces, providing an orthonormal angular-momentum eigenbasis for symmetric tensors of any rank. The relationship between the spherical-harmonic tensors and spin-weighted spherical harmonics is derived. The results facilitate the spherical-harmonic expansion of a large class of tensor-valued functions. Several simple illustrative examples are discussed, and the formalism is used to derive the leading-order effects of violations of Lorentz invariance in Newtonian gravity.
\end{abstract}

DOI: 10.1103/PhysRevResearch.2.043061

\section{INTRODUCTION}

Spherical harmonics $Y_{j m}$ provide an orthonormal basis for scalar functions on the 2-sphere and have numerous applications in physics and related fields. While they are commonly written in terms of the spherical-coordinate polar angle $\theta$ and azimuthal angle $\phi$, spherical harmonics can be expressed in terms of Cartesian coordinates, which is convenient in certain applications. The Cartesian versions involve rank- $j$ symmetric trace-free tensors $\mathcal{Y}_{j m}$ [1-5]. These form a basis for traceless tensors and provide a link between functions on the sphere and symmetric traceless tensors in three dimensions.

This work builds on the above understanding in several ways. We first develop a method for calculating the scalar spherical harmonics $Y_{j m}$ in terms of components of the direction unit vector

$$
\boldsymbol{n}=\sin \theta \cos \phi \boldsymbol{e}_{x}+\sin \theta \sin \phi \boldsymbol{e}_{y}+\cos \theta \boldsymbol{e}_{z} .
$$

The result can be used to write the spherical harmonics in terms of Cartesian coordinates, spherical-coordinate angles, or any other coordinates. We then extract the traceless $\mathcal{Y}_{j m}$ tensors and study their properties. These are extended to rank- $\varrho$ tensors $\mathcal{Y}_{j m}^{\varrho}$ with nonzero trace, which can be used to perform both a trace and angular-momentum decomposition of an arbitrary tensor. The formalism is then generalized to spin-weighted spherical harmonics ${ }_{s} Y_{j m}$ [6-8] and tensorvalued function spaces.

Spherical harmonics are eigenfunctions of angular momentum $\boldsymbol{J}=\boldsymbol{S}+\boldsymbol{L}$, with eigenvalues $\boldsymbol{J}^{2}=j(j+1)$ and $J_{z}=m$, where $m$ is limited by $|m| \leqslant j$. Angular momentum is the generator for rotations, so spherical harmonics provide a natural characterization of the rotational properties and direction

Published by the American Physical Society under the terms of the Creative Commons Attribution 4.0 International license. Further distribution of this work must maintain attribution to the author(s) and the published article's title, journal citation, and DOI. dependence of a system. For a scalar function $f(\boldsymbol{n})$, the spin $S$ is zero, and $\boldsymbol{J}$ is purely orbital angular momentum $\boldsymbol{L}$, which accounts for the functional dependence on $\boldsymbol{n}$. The spherical decomposition $f(\boldsymbol{n})=\sum_{j m} f_{j m} Y_{j m}(\boldsymbol{n})$ involves quantum numbers $\{j, m\}$ associated with the compatible operators $\left\{J^{2}, J_{z}\right\}=\left\{L^{2}, L_{z}\right\}$. Each term in the expansion represents just one example of a structure with definite $J^{2}$ and $J_{z}$.

In contrast to scalar functions, constant tensors are purespin objects with zero orbital angular momentum. Consider, for example, a constant traceless symmetric tensor $T$ of rank $\varrho$. In this case, the total spin and total angular momentum are both $j=\varrho$. The symmetry of $T$ implies a total of $2 j+1$ independent components, matching the number of $m$ values for fixed $j$. It can be expanded in spin-eigenbasis tensors $\mathcal{Y}_{j m}$, $T=\sum_{m} T_{m} \mathcal{Y}_{j m}$. Each term in this expansion has the same total angular momentum as the corresponding $Y_{j m}$ term in the expansion of the scalar $f(\boldsymbol{n})$, but in the form of spin rather than orbital angular momentum.

It is not surprising that a connection exists between spherical harmonics $Y_{j m}(\boldsymbol{n})$ and the $\mathcal{Y}_{j m}$ basis tensors. In fact, the contraction of the $\mathcal{Y}_{j m}$ tensor with the $\boldsymbol{n}$ vector $j$ times yields a scalar function proportional to $Y_{j m}(\boldsymbol{n})$. This provides a link between scalar functions and constant tensors, a relation that can be generalized to tensor-valued functions. Contracting $\mathcal{Y}_{j m}$ with a single $\boldsymbol{n}$ vector gives a traceless symmetric rank$(j-1)$ tensor function of $\boldsymbol{n}$. This decreases the spin by 1 and increases the orbital angular momentum by 1 , while leaving the total angular momentum unchanged. Subsequent contractions with $\boldsymbol{n}$ continue to convert spin angular momentum to orbital angular momentum until we arrive at the scalar spherical harmonics $Y_{j m}(\boldsymbol{n})$. Consequently, each $\mathcal{Y}_{j m}$ generates a set of $j+1$ tensor-valued eigenfunctions of $J^{2}$ and $J_{z}$ with different ranks. For example, the tensor $\mathcal{Y}_{30}$ generates four different angular-momentum eigenfunctions: the spin- 3 constant $\left(\mathcal{Y}_{30}\right)^{a b c}$, the spin-2 $\left(\mathcal{Y}_{30}\right)^{a b c} n_{c}$, the spin-1 $\left(\mathcal{Y}_{30}\right)^{a b c} n_{b} n_{c}$, and the scalar $\left(\mathcal{Y}_{30}\right)^{a b c} n_{a} n_{b} n_{c}$. This procedure yields a natural set of tensor spherical harmonics of different ranks and spins. The components of these tensors in a special helicity basis [9] are the spin-weighted spherical harmonics up to a normalization factor. 
This paper is organized as follows. The basic theory is given in Sec. II. Section II A establishes some notation and conventions. An expression for scalar spherical harmonics $Y_{j m}$ in terms of the components of $\boldsymbol{n}$ is derived in Sec. II B. This expression is used in Sec. II C to construct the traceless rank- $j$ spherical-harmonic tensors $\mathcal{Y}_{j m}$. Section II D extends the $\mathcal{Y}_{j m}$ to rank- $\varrho$ tensors $\mathcal{Y}_{j m}^{\varrho}$ with nonzero trace. The connection between the $\mathcal{Y}_{j m}^{\varrho}$ and spin-weighted spherical harmonics ${ }_{s} Y_{j m}$ is derived in Sec. II E. Some simple illustrative examples are given in Sec. III. An application involving Lorentz-invariance violation in Newtonian gravity is discussed in Sec. IV. Spin weight and spin-weighted spherical harmonics are reviewed in Appendix A. Appendix B provides a brief overview of Young symmetrizers.

\section{CONSTRUCTION}

\section{A. Notation and conventions}

This section establishes some basic notation used throughout this paper. First, Latin indices $a, b, c, \ldots$ on tensor components indicate spatial dimensions in one of the coordinate systems described below. Greek letters $\alpha, \beta, \gamma, \ldots$ are used in Sec. IV to indicate spacetime indices.

Several different special sets of basis vectors are useful. In addition to the Cartesian basis $\left\{\boldsymbol{e}_{x}, \boldsymbol{e}_{y}, \boldsymbol{e}_{z}\right\}=\left\{\boldsymbol{e}^{x}, \boldsymbol{e}^{y}, \boldsymbol{e}^{z}\right\}$, we define $J_{z}$-basis vectors $\left\{\boldsymbol{e}_{\uparrow}, \boldsymbol{e}_{\downarrow}, \boldsymbol{e}_{z}\right\}=\left\{\boldsymbol{e}^{\downarrow}, \boldsymbol{e}^{\uparrow}, \boldsymbol{e}^{z}\right\}$, where

$$
\boldsymbol{e}_{\uparrow}=\boldsymbol{e}^{\downarrow}=\frac{1}{\sqrt{2}}\left(\boldsymbol{e}_{x}+i \boldsymbol{e}_{y}\right), \quad \boldsymbol{e}_{\downarrow}=\boldsymbol{e}^{\uparrow}=\frac{1}{\sqrt{2}}\left(\boldsymbol{e}_{x}-i \boldsymbol{e}_{y}\right) .
$$

The standard spherical-coordinate basis vectors are denoted as $\left\{\boldsymbol{e}_{r}, \boldsymbol{e}_{\theta}, \boldsymbol{e}_{\phi}\right\}=\left\{\boldsymbol{e}^{r}, \boldsymbol{e}^{\theta}, \boldsymbol{e}^{\phi}\right\}$, where

$$
\begin{aligned}
& \boldsymbol{e}_{r}=\boldsymbol{n}, \quad \boldsymbol{e}_{\theta}=\cos \theta \cos \phi \boldsymbol{e}_{x}+\cos \theta \sin \phi \boldsymbol{e}_{y}-\sin \theta \boldsymbol{e}_{z}, \\
& \boldsymbol{e}_{\phi}=-\sin \phi \boldsymbol{e}_{x}+\cos \phi \boldsymbol{e}_{y} .
\end{aligned}
$$

Finally, we define a helicity basis $\left\{\boldsymbol{e}_{r}, \boldsymbol{e}_{+}, \boldsymbol{e}_{-}\right\}=\left\{\boldsymbol{e}^{r}, \boldsymbol{e}^{-}, \boldsymbol{e}^{+}\right\}$, where

$$
\boldsymbol{e}_{ \pm}=\boldsymbol{e}^{\mp}=\frac{1}{\sqrt{2}}\left(\boldsymbol{e}_{\theta} \pm i \boldsymbol{e}_{\phi}\right)
$$

Note that raising and lowering indices in the $J_{z}$ basis exchanges "up" and "down" labels, while raising and lowering indices in the helicity basis exchanges "plus" and "minus" labels. All bases are defined to be orthonormal: $\boldsymbol{e}_{a} \cdot \boldsymbol{e}^{b}=\delta_{a}^{b}$. We denote the direction cosines between two vectors, not necessarily from the same basis, as $g_{a a^{\prime}}=\boldsymbol{e}_{a} \cdot \boldsymbol{e}_{a^{\prime}}, g^{a a^{\prime}}=\boldsymbol{e}^{a} \cdot \boldsymbol{e}^{a^{\prime}}$, and $g_{a}^{a^{\prime}}=\boldsymbol{e}_{a} \cdot \boldsymbol{e}^{a^{\prime}}$. Note that these are the components of the Euclidean metric $g=g^{a a^{\prime}} \boldsymbol{e}_{a} \otimes \boldsymbol{e}_{a^{\prime}}$ relative to row basis $\boldsymbol{e}_{a}$ and column basis $\boldsymbol{e}_{a^{\prime}}$. In addition to defining the inner product, the metric components can be used to transform tensor components between bases, including the raising and lowering of indices.

We denote the symmetrized tensor product using $\odot$. The symmetrized product of two vectors $v$ and $u$ is defined to be $v \odot u=\frac{1}{2}(v \otimes u+u \otimes v)$. The $k$-fold symmetrized product of a vector $v$ will be written as $v^{\odot k}$, which in index notation reads $\left(v^{\odot k}\right)^{a_{1} \cdots a_{k}}=\frac{1}{k !} v^{\left(a_{1}\right.} \cdots v^{\left.a_{k}\right)}=v^{a_{1}} \cdots v^{a_{k}}$. This is the simple $k$-fold tensor product. The product of products is written $\left(v^{\odot k} \odot u^{\odot l}\right)^{a_{1} \cdots a_{k+l}}=\frac{1}{(k+l) !} v^{\left(a_{1}\right.} \cdots v^{a_{k}} u^{a_{k+1}} \cdots u^{\left.a_{k+l}\right)}$. More generally, the product of a rank- $k$ tensor $T$ and a rank- $l$ tensor $S$ is $(T \odot S)^{a_{1} \cdots a_{k+l}}=\frac{1}{(k+l) !} T^{\left(a_{1} \cdots a_{k}\right.} S^{\left.a_{k+1} \cdots a_{k+l}\right)}$. We write a $k$-fold symmetric product of a tensor $T$ as $T^{\odot k}$. The inner product of two equal-rank tensors $T$ and $S$ is defined as the invariant contraction $T \cdot S=T^{a_{1} \cdots a_{k}} S_{a_{1} \cdots a_{k}}$. Finally, a tensor index written with an exponent, such as $a^{q}$, indicates $q$ copies of the index $a$. For example, $T^{x^{2} y^{3} z}=T^{x x y y y z}$ is a Cartesianbasis component of a rank-6 tensor $T$.

\section{B. Scalar spherical harmonics}

In this section, we develop a method for calculating the scalar spherical harmonics $Y_{j m}$ in terms of the components of $\boldsymbol{n}$. The derivation favors the $J_{z}$ basis, in which the components of $\boldsymbol{n}$ are given by

$$
\begin{aligned}
& n_{\uparrow}=\frac{1}{\sqrt{2}}\left(n_{x}+i n_{y}\right)=\frac{1}{\sqrt{2}} \sin \theta e^{+i \phi}, \\
& n_{\downarrow}=\frac{1}{\sqrt{2}}\left(n_{x}-i n_{y}\right)=\frac{1}{\sqrt{2}} \sin \theta e^{-i \phi}, \quad n_{z}=\cos \theta .
\end{aligned}
$$

The result of the calculation that follows is

$$
\begin{aligned}
Y_{j m}(\boldsymbol{n})= & (-\operatorname{sgn} m)^{m} \sqrt{\frac{(2 j+1)(j+m) !(j-m) !}{4 \pi 2^{|m|}}} \\
& \times \sum_{\left.q\right|_{j m}} \frac{n_{\uparrow}^{q_{\uparrow}} n_{\downarrow}^{q_{\downarrow}} n_{z}^{q_{z}}}{(-2)^{\widehat{q}_{\uparrow} \downarrow} q_{\uparrow} ! q_{\downarrow} ! q_{z} !},
\end{aligned}
$$

where $\left.q\right|_{j m}$ is the restriction to all non-negative powers $q=\left\{q_{\uparrow}, q_{\downarrow}, q_{z}\right\}$ that sum to $j$ and obey $q_{\uparrow}-q_{\downarrow}=m$, and $\widehat{q}_{\uparrow \downarrow}=\min \left(q_{\uparrow}, q_{\downarrow}\right)$. In practice, this can be accomplished by summing over $q_{z}=j-|m|, j-|m|-2, j-|m|-4 \cdots \geqslant$ 0 , with the remaining powers set to $q_{\uparrow}=\frac{1}{2}\left(j+m-q_{z}\right)$ and $q_{\downarrow}=\frac{1}{2}\left(j-m-q_{z}\right)$. For illustrative purposes, $Y_{j m}$ up to $j=$ 4 are given in Table I. Combining Eqs. (5) and (6), we can write the spherical harmonics in terms of Cartesian components of $\boldsymbol{n}$. It also leads to

$$
\begin{aligned}
Y_{j m}(\theta, \phi)= & (-\operatorname{sgn} m)^{m} \sqrt{\frac{(2 j+1)(j+m) !(j-m) !}{4 \pi 2^{|m|}}} e^{i m \phi} \\
& \times \sum_{\left.q\right|_{j m}} \frac{(\sin \theta)^{q_{\uparrow}+q_{\downarrow}}(\cos \theta)^{q_{z}}}{2^{\left(q_{\uparrow}+q_{\downarrow}\right) / 2}(-2)^{q_{\uparrow \downarrow}} q_{\uparrow} ! q_{\downarrow} ! q_{z} !},
\end{aligned}
$$

in terms of the spherical-coordinate angles.

The derivation of Eq. (6) starts by taking $s_{1}=s_{2}=0, j_{1}=$ $1, m_{1}= \pm 1$, and $m_{2}= \pm j_{2}$ in identity (A5). This yields the recursion relation

$$
Y_{j( \pm j)}(\boldsymbol{n})=\sqrt{\frac{2 j+1}{j}}\left\{\begin{array}{c}
-n_{\uparrow} \\
n_{\downarrow}
\end{array}\right\} Y_{(j-1)( \pm j \mp 1)}(\boldsymbol{n}),
$$

which relates different harmonics at the upper and lower limits of $m= \pm j$. Combining this with $Y_{00}=1 / \sqrt{4 \pi}$, we then find

$$
Y_{j( \pm j)}(\boldsymbol{n})=\sqrt{\frac{(2 j+1) ! !}{4 \pi j !}}\left\{\begin{array}{c}
\left(-n_{\uparrow}\right)^{j} \\
\left(n_{\downarrow}\right)^{j}
\end{array}\right\} .
$$

We next use ladder operators to find the harmonics for other values of $m$.

The ladder operators $J_{\uparrow}=\boldsymbol{e}_{\uparrow} \cdot \boldsymbol{J}$ and $J_{\downarrow}=\boldsymbol{e}_{\downarrow} \cdot \boldsymbol{J}$ can be used, respectively, to raise and lower the $J_{z}$-eigenvalue $m$. When acting on spin-zero scalars, the ladder operators can be 
TABLE I. Spherical harmonics and the traceless spherical-harmonic tensors for $j \leqslant 4$. Only the non-negative $m$ cases are shown. The $Y_{j m}$ for negative $m$ can be found using $Y_{j(-m)}=(-1)^{m} Y_{j m}^{*}$, which results in the replacement $n_{\uparrow} \leftrightarrow n_{\downarrow}$ and multiplication by the Condon-Shortley phase $(-1)^{m}$. The $\mathcal{Y}_{j m}$ for negative $m$ can be found using $\mathcal{Y}_{j(-m)}=(-1)^{m} \mathcal{Y}_{j m}^{*}$, resulting in the replacement $\boldsymbol{e}_{\uparrow} \leftrightarrow \boldsymbol{e}_{\downarrow}$ and multiplication by $(-1)^{m}$.

\begin{tabular}{|c|c|c|}
\hline$j m$ & $Y_{j m}(\boldsymbol{n} 2)$ & $\mathcal{Y}_{j m}$ \\
\hline 00 & $\sqrt{\frac{1}{4 \pi}}$ & 1 \\
\hline 10 & $\sqrt{\frac{3}{4 \pi}} n_{z}$ & $\boldsymbol{e}_{z}$ \\
\hline 11 & $-\sqrt{\frac{3}{4 \pi}} n_{\uparrow}$ & $-\boldsymbol{e}_{\uparrow}$ \\
\hline 20 & $\sqrt{\frac{5}{4 \pi}}\left(n_{z}^{2}-n_{\uparrow} n_{\downarrow}\right)$ & $\sqrt{\frac{2}{3}}\left(\boldsymbol{e}_{z} \odot \boldsymbol{e}_{z}-\boldsymbol{e}_{\uparrow} \odot \boldsymbol{e}_{\downarrow}\right)$ \\
\hline 21 & $-\sqrt{\frac{15}{4 \pi}} n_{\uparrow} n_{z}$ & $-\sqrt{2} \boldsymbol{e}_{\uparrow} \odot \boldsymbol{e}_{z}$ \\
\hline 22 & $\sqrt{\frac{15}{8 \pi}} n_{\uparrow}^{2}$ & $\boldsymbol{e}_{\uparrow} \odot \boldsymbol{e}_{\uparrow}$ \\
\hline 30 & $\sqrt{\frac{7}{4 \pi}}\left(n_{z}^{3}-3 n_{\uparrow} n_{\downarrow} n_{z}\right)$ & $\sqrt{\frac{2}{5}}\left(\boldsymbol{e}_{z} \odot \boldsymbol{e}_{z} \odot \boldsymbol{e}_{z}-3 \boldsymbol{e}_{\uparrow} \odot \boldsymbol{e}_{\downarrow} \odot \boldsymbol{e}_{z}\right)$ \\
\hline 31 & $-\sqrt{\frac{21}{8 \pi}}\left(2 n_{\uparrow} n_{z}^{2}-n_{\uparrow}^{2} n_{\downarrow}\right)$ & $-\sqrt{\frac{3}{5}}\left(2 \boldsymbol{e}_{\uparrow} \odot \boldsymbol{e}_{z} \odot \boldsymbol{e}_{z}-\boldsymbol{e}_{\uparrow} \odot \boldsymbol{e}_{\uparrow} \odot \boldsymbol{e}_{\downarrow}\right)$ \\
\hline 32 & $\sqrt{\frac{105}{8 \pi}} n_{\uparrow}^{2} n_{z}$ & $\sqrt{3} \boldsymbol{e}_{\uparrow} \odot \boldsymbol{e}_{\uparrow} \odot \boldsymbol{e}_{z}$ \\
\hline 33 & $-\sqrt{\frac{35}{8 \pi}} n_{\uparrow}^{3}$ & $-\boldsymbol{e}_{\uparrow} \odot \boldsymbol{e}_{\uparrow} \odot \boldsymbol{e}_{\uparrow}$ \\
\hline 40 & $\sqrt{\frac{9}{16 \pi}}\left(2 n_{z}^{4}-12 n_{\uparrow} n_{\downarrow} n_{z}^{2}+3 n_{\uparrow}^{2} n_{\downarrow}^{2}\right)$ & $\sqrt{\frac{2}{35}}\left(2 \boldsymbol{e}_{z} \odot \boldsymbol{e}_{z} \odot \boldsymbol{e}_{z} \odot \boldsymbol{e}_{z}-12 \boldsymbol{e}_{\uparrow} \odot \boldsymbol{e}_{\downarrow} \odot \boldsymbol{e}_{z} \odot \boldsymbol{e}_{z}+3 \boldsymbol{e}_{\uparrow} \odot \boldsymbol{e}_{\uparrow} \odot \boldsymbol{e}_{\downarrow} \odot \boldsymbol{e}_{\downarrow}\right)$ \\
\hline 41 & $-\sqrt{\frac{45}{8 \pi}}\left(2 n_{\uparrow} n_{z}^{3}-3 n_{\uparrow}^{2} n_{\downarrow} n_{z}\right)$ & $-\sqrt{\frac{4}{7}}\left(2 \boldsymbol{e}_{\uparrow} \odot \boldsymbol{e}_{z} \odot \boldsymbol{e}_{z} \odot \boldsymbol{e}_{z}-3 \boldsymbol{e}_{\uparrow} \odot \boldsymbol{e}_{\uparrow} \odot \boldsymbol{e}_{\downarrow} \odot \boldsymbol{e}_{z}\right)$ \\
\hline 42 & $\sqrt{\frac{45}{8 \pi}}\left(3 n_{\uparrow}^{2} n_{z}^{2}-n_{\uparrow}^{3} n_{\downarrow}\right)$ & $\sqrt{\frac{4}{7}}\left(3 \boldsymbol{e}_{\uparrow} \odot \boldsymbol{e}_{\uparrow} \odot \boldsymbol{e}_{z} \odot \boldsymbol{e}_{z}-\boldsymbol{e}_{\uparrow} \odot \boldsymbol{e}_{\uparrow} \odot \boldsymbol{e}_{\uparrow} \odot \boldsymbol{e}_{\downarrow}\right)$ \\
\hline 43 & $-\sqrt{\frac{315}{8 \pi}} n_{\uparrow}^{3} n_{z}$ & $-2 \boldsymbol{e}_{\uparrow} \odot \boldsymbol{e}_{\uparrow} \odot \boldsymbol{e}_{\uparrow} \odot \boldsymbol{e}_{z}$ \\
\hline 44 & $\sqrt{\frac{315}{32 \pi}} n_{\uparrow}^{4}$ & $\boldsymbol{e}_{\uparrow} \odot \boldsymbol{e}_{\uparrow} \odot \boldsymbol{e}_{\uparrow} \odot \boldsymbol{e}_{\uparrow}$ \\
\hline
\end{tabular}

written as the differential operators $J_{\uparrow}=\frac{e^{i \phi}}{\sqrt{2}}\left(\partial_{\theta}+i \cot \theta \partial_{\phi}\right)$ and $J_{\downarrow}=\frac{e^{-i \phi}}{\sqrt{2}}\left(-\partial_{\theta}+i \cot \theta \partial_{\phi}\right)$. Acting on the $J_{z}$-basis components of $\boldsymbol{n}$, the ladder operators shift the components according to $J_{\uparrow} n_{\uparrow}=-J_{\downarrow} n_{\downarrow}=0, J_{\uparrow} n_{\downarrow}=-J_{\downarrow} n_{\uparrow}=n_{z}, J_{\uparrow} n_{z}=$ $-n_{\uparrow}$, and $J_{\downarrow} n_{z}=n_{\downarrow}$. As a result, repeatedly operating on Eq. (9) with $J_{\downarrow}$ or $J_{\uparrow}$ introduces other components of $\boldsymbol{n}$, leaving the total number of components appearing in the product unchanged. The $Y_{j m}$ harmonics are then combinations of terms involving $n_{\uparrow}^{q_{\uparrow}} n_{\downarrow}^{q_{\downarrow}} n_{z}^{q_{z}}$, with powers $q_{\uparrow}, q_{\downarrow}$, and $q_{z}$ that sum to $j$. Noting that $J_{z}=\boldsymbol{e}_{z} \cdot \boldsymbol{J}=-i \partial_{\phi}$, we then have $J_{z} n_{\uparrow}=$ $n_{\uparrow}, J_{z} n_{\downarrow}=-n_{\downarrow}$, and $J_{z} n_{z}=0$, which gives $J_{z} n_{\uparrow}^{q_{\uparrow}} n_{\downarrow}^{q_{\downarrow}} n_{z}^{q_{z}}=$ $\left(q_{\uparrow}-q_{\downarrow}\right) n_{\uparrow}^{q_{\uparrow}} n_{\downarrow}^{q_{\downarrow}} n_{z}^{q_{z}}$. So the powers also obey $q_{\uparrow}-q_{\downarrow}=m$. This implies that $q_{z}$ is restricted to $j-|m|, j-|m|-2, j-$ $|m|-4 \cdots \geqslant 0$, with the remaining powers given by $q_{\uparrow}=$ $\frac{1}{2}\left(j+m-q_{z}\right)$ and $q_{\downarrow}=\frac{1}{2}\left(j-m-q_{z}\right)$. The harmonics then take the form

$$
Y_{j m}(\boldsymbol{n})=\sum_{q_{z}} A_{j m}^{q_{z}} n_{\uparrow}^{\frac{1}{2}\left(j+m-q_{z}\right)} n_{\downarrow}^{\frac{1}{2}\left(j-m-q_{z}\right)} n_{z}^{q_{z}},
$$

where the constant coefficients $A_{j m}^{q_{z}}$ are nonzero for the values of $q_{z}$ given above.

Equation (9) implies that the nonzero coefficients for $m=$ $\pm j$ are $A_{j( \pm j)}^{0}=(\mp 1)^{j} \sqrt{(2 j+1) ! ! / 4 \pi j !}$. To find the other
$A_{j m}^{q_{z}}$ coefficients, we adopt the conventional normalization

$$
\left\{\begin{array}{l}
J_{\uparrow} \\
J_{\downarrow}
\end{array}\right\} Y_{j m}=\sqrt{\frac{1}{2}(j \pm m+1)(j \mp m)} Y_{j(m \pm 1)} .
$$

Ladder operations then lead to the recursion relation

$$
\begin{aligned}
& \sqrt{\frac{1}{2}(j \pm m+1)(j \mp m)} A_{j(m \pm 1)}^{q_{z}} \\
& \quad= \pm \frac{1}{2}\left(j \mp m-q_{z}+1\right) A_{j m}^{q_{z}-1} \mp\left(q_{z}+1\right) A_{j m}^{q_{z}+1} .
\end{aligned}
$$

By replacing $m \rightarrow \mp(|m|+1)$ and taking $q_{z}=j-|m|$, we get a recursion for cases in which either $q_{\uparrow}$ or $q_{\downarrow}$ vanishes, which leads to

$$
A_{j m}^{j-|m|}=\frac{(-\operatorname{sgn} m)^{m}}{|m| !} \sqrt{\frac{(2 j+1)}{4 \pi 2^{|m|}} \frac{(j+|m|) !}{(j-|m|) !}} .
$$

We find a closed-form expression for the remaining coefficients by combining the raising and lowering relations to get a recursion between coefficients with the same $m$ :

$$
\begin{aligned}
0= & \left(q_{z}+1\right)\left(q_{z}+2\right) A_{j m}^{q_{z}+2} \\
& +\frac{\left(j+m-q_{z}\right)\left(j-m-q_{z}\right)+\left(q_{z}-1\right) q_{z}}{2} A_{j m}^{q_{z}} \\
& +\frac{\left(j+m-q_{z}+2\right)\left(j-m-q_{z}+2\right)}{4} A_{j m}^{q_{z}-2} .
\end{aligned}
$$


Defining

$$
\begin{aligned}
B_{j m}^{q_{z}}= & \left(q_{z}+1\right)\left(q_{z}+2\right) A_{j m}^{q_{z}+2} \\
& +\frac{\left(j+m-q_{z}\right)\left(j-m-q_{z}\right)}{2} A_{j m}^{q_{z}},
\end{aligned}
$$

the recursion relation can be written as $B_{j m}^{q_{z}-2}=-2 B_{j m}^{q_{z}}$. This implies that all the $B_{j m}^{q_{z}}$ constants are proportional to $B_{j m}^{j-|m|}$, which is zero. Therefore, all $B_{j m}^{q_{z}}$ constants vanish, and we have

$$
A_{j m}^{q_{z}}=-\frac{2\left(q_{z}+1\right)\left(q_{z}+2\right)}{\left(j+m-q_{z}\right)\left(j-m-q_{z}\right)} A_{j m}^{q_{z}+2}
$$

With this we can write all of the coefficients in terms of those given in Eq. (13). The result reduces to

$$
A_{j m}^{q_{z}}=\frac{(-\operatorname{sgn} m)^{m}}{(-2)^{\widehat{q_{\uparrow} \downarrow} q_{\uparrow} ! q_{\downarrow} ! q_{z} !}} \sqrt{\frac{(2 j+1)(j+m) !(j-m) !}{4 \pi 2^{|m|}}},
$$

where $q_{\uparrow}=\frac{1}{2}\left(j+m-q_{z}\right), q_{\downarrow}=\frac{1}{2}\left(j-m-q_{z}\right)$, and $\widehat{q}_{\uparrow \downarrow}=$ $\min \left(q_{\uparrow}, q_{\downarrow}\right)$. We then arrive at Eq. (6).

\section{Traceless spherical-harmonic tensors}

Next, we extract the orthonormal rank- $j$ symmetric traceless tensors $\mathcal{Y}_{j m}$ and discuss their properties. Notice that Eq. (6) can be written as the inner product of two rank-j tensors,

$$
Y_{j m}(\boldsymbol{n})=\sqrt{\frac{(2 j+1) ! !}{4 \pi j !}} \mathcal{Y}_{j m} \cdot \boldsymbol{n}^{\odot j},
$$

where the spherical-harmonic tensors are defined as

$$
\begin{aligned}
\mathcal{Y}_{j m}= & (-\operatorname{sgn} m)^{m} \sqrt{\frac{j !(j+m) !(j-m) !}{2^{|m|}(2 j-1) ! !}} \\
& \times \sum_{\left.q\right|_{j m}} \frac{\boldsymbol{e}_{\uparrow}^{\odot q_{\uparrow}} \odot \boldsymbol{e}_{\downarrow}^{\odot q_{\downarrow}} \odot \boldsymbol{e}_{z}^{\odot q_{z}}}{(-2)^{\widehat{q_{\uparrow} \downarrow} q_{\uparrow} ! q_{\downarrow} ! q_{z} !}} .
\end{aligned}
$$

Examples of spherical-harmonic tensors for $j \leqslant 4$ are included in Table I. While these are conveniently expressed in terms of the $J_{z}$-basis vectors, they can be written in the Cartesian basis using Eq. (2). More generally, the components of $\mathcal{Y}_{j m}$ in any basis $\boldsymbol{e}_{a}$ can be written in terms of the direction cosines between the $\boldsymbol{e}_{a}$ vectors and the $J_{z}$-basis vectors:

$$
\begin{aligned}
& \left(\mathcal{Y}_{j m}\right)^{a_{1} a_{2} \cdots a_{j}} \\
& =\mathcal{Y}_{j m} \cdot\left(\boldsymbol{e}^{a_{1}} \otimes \boldsymbol{e}^{a_{2}} \otimes \cdots \otimes \boldsymbol{e}^{a_{j}}\right) \\
& =(-\operatorname{sgn} m)^{m} \sqrt{\frac{(j+m) !(j-m) !}{2^{|m|} j !(2 j-1) ! !}} \\
& \quad \times \sum_{q \mid j m} \frac{g_{\uparrow}^{\left(a_{1}\right.} \cdots g_{\uparrow}^{a_{q_{\uparrow}}} g_{\downarrow}^{a_{q_{\uparrow}+1}} \cdots g_{\downarrow}^{a_{q_{\uparrow}+q_{\downarrow}}} g_{z}^{a_{q_{\uparrow}+q_{\downarrow}+1}} \cdots g_{z}^{\left.a_{j}\right)}}{(-2)^{\widehat{\widehat{q}_{\uparrow}}} q_{\uparrow} ! q_{\downarrow} ! q_{z} !} .
\end{aligned}
$$

The complex conjugate of $\mathcal{Y}_{j m}$ is given by

$$
\begin{aligned}
\mathcal{Y}_{j m}^{*}= & (-\operatorname{sgn} m)^{m} \sqrt{\frac{j !(j+m) !(j-m) !}{2^{|m|}(2 j-1) ! !}} \\
& \times \sum_{\left.q\right|_{j m}} \frac{\boldsymbol{e}^{\uparrow \odot q_{\uparrow}} \odot \boldsymbol{e}^{\downarrow \odot q_{\downarrow}} \odot \boldsymbol{e}^{z \odot q_{z}}}{(-2)^{\widehat{q}_{\uparrow \downarrow} q_{\uparrow} ! q_{\downarrow} ! q_{z} !} .}
\end{aligned}
$$

Note that the $\mathcal{Y}_{j m}$ include the Condon-Shortley phase and obey the relation

$$
\mathcal{Y}_{j m}^{*}=(-1)^{m} \mathcal{Y}_{j(-m)}
$$

Below we show that the spherical-harmonic tensors obey the orthonormality relation

$$
\mathcal{Y}_{j m} \cdot \mathcal{Y}_{j m^{\prime}}^{*}=\delta_{m m^{\prime}}
$$

and serve as an orthonormal basis for the $(2 j+1)$ dimensional space of rank- $j$ symmetric traceless tensors in three dimensions.

Using the $\mathcal{Y}_{j m}$, we can perform a spherical decomposition of an arbitrary symmetric traceless rank- $j$ tensor $T$,

$$
T=\sum_{m} T_{j m} \mathcal{Y}_{j m}
$$

The spherical-expansion coefficients are given by the inner product with the conjugate basis tensor,

$$
\begin{aligned}
T_{j m}=\mathcal{Y}_{j m}^{*} \cdot T= & (-\operatorname{sgn} m)^{m} \sqrt{\frac{j !(j+m) !(j-m) !}{2^{|m|}(2 j-1) ! !}} \\
& \times \sum_{\left.q\right|_{j m}} \frac{T^{\uparrow^{q_{\uparrow}} \downarrow^{q_{\downarrow}} z^{q_{z}}}}{(-2)^{\widehat{q_{\uparrow} \downarrow}} q_{\uparrow} ! q_{\downarrow} ! q_{z} !} .
\end{aligned}
$$

The $T$ tensor and the basis tensors $\mathcal{Y}_{j m}$ are spin- $j$ objects. Each component has the same $j$ value but can have different $m$ values. The $T_{j m}$ give the components with fixed $J_{z}=m$.

The remainder of this section is devoted to proving that the $\mathcal{Y}_{j m}$ are traceless and orthonormal. To show that they are traceless, we first note that the trace of $\boldsymbol{e}_{\uparrow}^{\odot q_{\uparrow}} \odot \boldsymbol{e}_{\downarrow}^{\odot q_{\downarrow}} \odot \boldsymbol{e}_{z}^{\odot q_{z}}$ is

$$
\begin{aligned}
& \frac{q_{z}\left(q_{z}-1\right)}{j(j-1)} \boldsymbol{e}_{\uparrow}^{\odot q_{\uparrow}} \odot \boldsymbol{e}_{\downarrow}^{\odot q_{\downarrow}} \odot \boldsymbol{e}_{z}^{\odot\left(q_{z}-2\right)} \\
& \quad+\frac{2 q_{\uparrow} q_{\downarrow}}{j(j-1)} \boldsymbol{e}_{\uparrow}^{\odot\left(q_{\uparrow}-1\right)} \odot \boldsymbol{e}_{\downarrow}^{\odot\left(q_{\downarrow}-1\right)} \odot \boldsymbol{e}_{z}^{\odot q_{z}} .
\end{aligned}
$$

So the trace of Eq. (19) is proportional to

$$
\begin{aligned}
& \sum_{\left.q\right|_{j m}} \frac{\boldsymbol{e}_{\uparrow}^{\odot q_{\uparrow}} \odot \boldsymbol{e}_{\downarrow}^{\odot q_{\downarrow}} \odot \boldsymbol{e}_{z}^{\odot\left(q_{z}-2\right)}}{(-2)^{\widehat{q}_{\uparrow \downarrow} q_{\uparrow} ! q_{\downarrow} !\left(q_{z}-2\right) !}} \\
& \quad+2 \sum_{q_{l_{j m}}} \frac{\boldsymbol{e}_{\uparrow}^{\odot\left(q_{\uparrow}-1\right)} \odot \boldsymbol{e}_{\downarrow}^{\odot\left(q_{\downarrow}-1\right)} \odot \boldsymbol{e}_{z}^{\odot q_{z}}}{(-2)^{\widehat{q_{\uparrow \downarrow} \downarrow}\left(q_{\uparrow}-1\right) !\left(q_{\downarrow}-1\right) ! q_{z} !}}=0,
\end{aligned}
$$

proving that the $\mathcal{Y}_{j m}$ tensors are traceless.

To show orthonormality, we contract $\mathcal{Y}_{j m}$ with $\mathcal{Y}_{j m^{\prime}}^{*}$. The orthogonality of the $\left\{\boldsymbol{e}_{\uparrow}, \boldsymbol{e}_{\downarrow}, \boldsymbol{e}_{z}\right\}$ basis implies that the only nonzero terms in the resulting double sum are those with matching $q$ powers. This immediately implies $\mathcal{Y}_{j m}$ tensors with different $m$ values are orthogonal. A short calculation 
then shows that the inner product of two $\mathcal{Y}_{j m}$ tensors reduces to

$$
\mathcal{Y}_{j m} \cdot \mathcal{Y}_{j m^{\prime}}^{*}=\delta_{m m^{\prime}} \frac{(j+m) !(j-m) !}{2^{|m|}(2 j-1) ! !} \sum_{\left.q\right|_{j m}} \frac{1}{4 \widehat{q}_{\uparrow \downarrow} q_{\uparrow} ! q_{\downarrow} ! q_{z} !} .
$$

It is then useful to relabel $q_{1}=\widehat{q}_{\uparrow \downarrow}=\min \left(q_{\uparrow}, q_{\downarrow}\right), q_{2}=$ $\max \left(q_{\uparrow}, q_{\downarrow}\right)$, and $q_{3}=q_{z}$. The sum in the above expression can be written as $\sum_{\left.q\right|_{j m}} \frac{1}{q_{1} ! q_{2} ! q_{3} !}\left(\frac{1}{4}\right)^{q_{1}}$ and is restricted to $q_{1}+q_{2}+q_{3}=j$ and $q_{2}-q_{1}=|m|$. It can be evaluated by considering the multinomial expansion

$$
\begin{aligned}
\frac{1}{j !}\left(\frac{1}{4 z}+z+1\right)^{j} & =\sum_{\left.q\right|_{j}} \frac{1}{q_{1} ! q_{2} ! q_{3} !}\left(\frac{1}{4}\right)^{q_{1}} z^{q_{2}-q_{1}} \\
& =\sum_{m=-j}^{j} C_{m} z^{m}
\end{aligned}
$$

where $\left.q\right|_{j}$ is the restriction to sets of non-negative powers $\left\{q_{1}, q_{2}, q_{3}\right\}$ adding to $j$. The $C_{m}$ expansion coefficients are partial sums further restricted by $q_{2}-q_{1}=m$. This implies that the sum in Eq. (28) is equivalent to the coefficient $C_{|m|}$. We can calculate the $C_{m}$ coefficients using the contour integral

$$
\begin{aligned}
C_{m} & =\frac{1}{2 \pi i} \oint_{\gamma} \frac{1}{j !}\left(\frac{1}{4 z}+z+1\right)^{j} z^{-(m+1)} d z \\
& =\frac{2^{m}(2 j-1) ! !}{(j+m) !(j-m) !},
\end{aligned}
$$

where $\gamma$ is any counterclockwise contour enclosing the origin in the complex plane. Along with Eq. (28), this result implies the $\mathcal{Y}_{j m}$ tensors are orthonormal.

\section{Generalized spherical-harmonic tensors}

By taking symmetric products of the $\mathcal{Y}_{j m}$ tensors with the metric $g$, we can generalize the $\mathcal{Y}_{j m}$ to create a basis for symmetric rank- $\varrho$ tensors including traces. Each metric increases the rank by 2 , so the number of metric tensors in the product is $\frac{1}{2}(\varrho-j)$. We then define

$$
\begin{aligned}
\mathcal{Y}_{j m}^{\varrho} & =\sqrt{\frac{\varrho !(2 j+1) ! !}{j !(\varrho+j+1) ! !(\varrho-j) ! !}} \mathcal{Y}_{j m} \odot g^{\odot \frac{1}{2}(\varrho-j)} \\
& =(-\operatorname{sgn} m)^{m} \sqrt{\frac{(2 j+1) \varrho !(j+m) !(j-m) !}{2^{|m|}(\varrho+j+1) ! !(\varrho-j) ! !}} \sum_{\left.q\right|_{j m}} \frac{\boldsymbol{e}_{\uparrow}^{\odot q_{\uparrow}} \odot \boldsymbol{e}_{\downarrow}^{\odot q_{\downarrow}} \odot \boldsymbol{e}_{z}^{\odot q_{z}} \odot g^{\odot \frac{1}{2}(\varrho-j)}}{(-2)^{\widehat{q} \uparrow \downarrow} q_{\uparrow} ! q_{\downarrow} ! q_{z} !}
\end{aligned}
$$

These form an orthonormal basis for symmetric rank- $\varrho$ tensors including trace elements. The $j m$ indices give the spin angular momentum of the basis tensor. The components in any basis $\boldsymbol{e}_{a}$ are given by

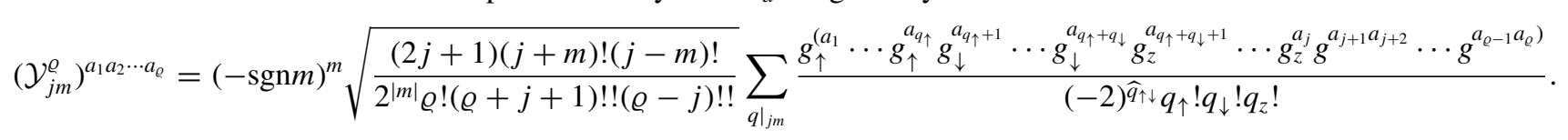

In terms of the rank- $\varrho$ spherical-harmonic tensors, the scalar spherical harmonics are

$$
Y_{j m}(\boldsymbol{n})=\sqrt{\frac{(\varrho+j+1) ! !(\varrho-j) ! !}{4 \pi \varrho !}} \mathcal{Y}_{j m}^{\varrho} \cdot \boldsymbol{n}^{\odot \varrho},
$$

providing a generalization of Eq. (18). The conjugate tensors are

$$
\mathcal{Y}_{j m}^{\varrho *}=(-\operatorname{sgn} m)^{m} \sqrt{\frac{(2 j+1) \varrho !(j+m) !(j-m) !}{2^{|m|}(\varrho+j+1) ! !(\varrho-j) ! !}} \sum_{q \mid j m} \frac{\boldsymbol{e}^{\uparrow \odot q_{\uparrow}} \odot \boldsymbol{e}^{\downarrow \odot q_{\downarrow}} \odot \boldsymbol{e}^{z \odot q_{z}} \odot g^{\odot \frac{1}{2}(\varrho-j)}}{(-2)^{\widehat{q_{\uparrow \downarrow}}} q_{\uparrow} ! q_{\downarrow} ! q_{z} !}
$$

and satisfy

$$
\mathcal{Y}_{j m}^{\varrho *}=(-1)^{m} \mathcal{Y}_{j(-m)}^{\varrho} .
$$

The $\mathcal{Y}_{j m}^{\varrho}$ of the same rank are orthonormal,

$$
\mathcal{Y}_{j m}^{\varrho} \cdot \mathcal{Y}_{j^{\prime} m^{\prime}}^{\varrho *}=\delta_{j j^{\prime}} \delta_{m m^{\prime}},
$$

which we prove below. Note that for fixed $\varrho$, the values of $j$ are restricted to $j=\varrho, \varrho-2, \varrho-4, \ldots \geqslant 0$, and the total number of $\mathcal{Y}_{j m}^{\varrho}$ tensors is $\sum(2 j+1)=\frac{1}{2}(\varrho+1)(\varrho+$ $2)$, matching the dimension of the space of symmetric rank- $\varrho$ tensors. We recover the traceless spherical-harmonic tensors $\mathcal{Y}_{j m}=\mathcal{Y}_{j m}^{j}$ when $j=\varrho$. For even $\varrho, j=m=0$ corresponds to the normalized total-trace element $\mathcal{Y}_{00}^{\varrho}=g \odot \cdots \odot$ $g / \sqrt{\varrho+1}$.

Any symmetric rank- $\varrho$ tensor $T$ can be expanded in the generalized spherical-harmonic tensors,

$$
T=\sum_{j m} T_{j m} \mathcal{Y}_{j m}^{\varrho}
$$


where the spherical components are

$$
\begin{aligned}
T_{j m}=\mathcal{Y}_{j m}^{\varrho *} \cdot T= & (-\operatorname{sgn} m)^{m} \sqrt{\frac{(2 j+1) \varrho !(j+m) !(j-m) !}{2^{|m|}(\varrho+j+1) ! !(\varrho-j) ! !}} \\
& \times \sum_{\left.q\right|_{j m}} \frac{T^{\uparrow^{q_{\uparrow}} \downarrow^{q_{\downarrow}} z^{q_{z}} a_{1} \ldots a_{(\varrho-j) / 2}} a_{1} \ldots a_{(\varrho-j) / 2}}{(-2)^{\widehat{q_{\uparrow} \downarrow} q_{\uparrow} ! q_{\downarrow} ! q_{z} !}}
\end{aligned}
$$

This provides both an angular-momentum decomposition and a trace decomposition. The trace decomposition can be written

$$
T=\sum_{j} \sqrt{\frac{\varrho !(2 j+1) ! !}{j !(\varrho+j+1) ! !(\varrho-j) ! !}} T_{j} \odot g^{\odot \frac{1}{2}(\varrho-j)},
$$

where

$$
T_{j}=\sum_{m} T_{j m} \mathcal{Y}_{j m}
$$

are rank- $j$, symmetric, and traceless.

Definition (31) implies that the generalized sphericalharmonic tensors of different rank are related through the recursion relation

$$
\mathcal{Y}_{j m}^{\varrho}=\sqrt{\frac{\varrho(\varrho-1)}{(\varrho+j+1)(\varrho-j)}} g \odot \mathcal{Y}_{j m}^{\varrho-2} .
$$

They are also connected by the trace identity

$$
g \cdot \mathcal{Y}_{j m}^{\varrho}=\sqrt{\frac{(\varrho+j+1)(\varrho-j)}{\varrho(\varrho-1)}} \mathcal{Y}_{j m}^{\varrho-2},
$$

where the dot - indicates the contraction of $g$ with any two indices of $\mathcal{Y}_{j m}^{\varrho}$, yielding a tensor of rank $\varrho-2$.

We prove trace relation (42) by first considering the contraction of the metric $g$ with the symmetric product $g \odot T$, where $T$ is a symmetric rank- $\varrho$ tensor. A calculation yields the identity

$$
g \cdot(g \odot T)=D_{\varrho} T+E_{\varrho} g \odot(g \cdot T),
$$

where $D_{\varrho}=2(2 \varrho+3) /(\varrho+2)(\varrho+1)$ and $E_{\varrho}=\varrho(\varrho-$ $1) /(\varrho+2)(\varrho+1)$. For fixed $j$ and $m$, we apply this to the tensors

$$
\begin{aligned}
T_{\varrho} & =g \odot T_{\varrho-2}=g^{\odot \frac{1}{2}(\varrho-j)} \odot \mathcal{Y}_{j m} \\
& =\sqrt{\frac{j !(\varrho+j+1) ! !(\varrho-j) ! !}{\varrho !(2 j+1) ! !}} \mathcal{Y}_{j m}^{\varrho},
\end{aligned}
$$

which gives

$$
g \cdot T_{\varrho}=g \cdot\left(g \odot T_{\varrho-2}\right)=D_{\varrho-2} T_{\varrho-2}+E_{\varrho-2} g \odot\left(g \cdot T_{\varrho-2}\right) .
$$

Iterating, we find

$$
\begin{aligned}
g \cdot T_{\varrho}= & \left(D_{\varrho-2}+E_{\varrho-2} D_{\varrho-4}+E_{\varrho-2} E_{\varrho-4} D_{\varrho-6}+\cdots\right. \\
& \left.+E_{\varrho-2} \cdots E_{j+2} D_{j}\right) T_{\varrho-2} \\
= & \frac{(\varrho+j+1)(\varrho-j)}{\varrho(\varrho-1)} T_{\varrho-2},
\end{aligned}
$$

which leads to Eq. (42).

Next, identities (41) and (42) can be used to show that the $\mathcal{Y}_{j m}^{\varrho}$ tensors are orthonormal. We first note that orthogonality follows immediately from the tracelessness and orthogonality of the $\mathcal{Y}_{j m}$. The inner product $\mathcal{Y}_{j m}^{\varrho} \cdot \mathcal{Y}_{j^{\prime} m^{\prime}}^{\varrho *}$ involves traces of $\mathcal{Y}_{j m}$ unless $j=j^{\prime}$ and is proportional to $\mathcal{Y}_{j m} \cdot \mathcal{Y}_{j m^{\prime}}^{*}=\delta_{m m^{\prime}}$ when $j=j^{\prime}$. Then using Eqs. (41) and (42), we can write the inner product as

$$
\begin{aligned}
\mathcal{Y}_{j m}^{\varrho} \cdot \mathcal{Y}_{j m}^{\varrho *} & =\sqrt{\frac{\varrho(\varrho-1)}{(\varrho+j+1)(\varrho-j)}}\left(g \odot \mathcal{Y}_{j m}^{(\varrho-2)}\right) \cdot \mathcal{Y}_{j m}^{\varrho *} \\
& =\sqrt{\frac{\varrho(\varrho-1)}{(\varrho+j+1)(\varrho-j)}} \mathcal{Y}_{j m}^{(\varrho-2)} \cdot\left(g \cdot \mathcal{Y}_{j m}^{\varrho *}\right) \\
& =\mathcal{Y}_{j m}^{(\varrho-2)} \cdot \mathcal{Y}_{j m}^{\varrho-2 *},
\end{aligned}
$$

which implies all $\mathcal{Y}_{j m}^{\varrho}$ are normalized since the lowest-rank $\mathcal{Y}_{j m}^{j}=\mathcal{Y}_{j m}$ are normalized.

\section{E. Spin-weighted spherical harmonics}

The goal of this section is to show that each helicitybasis component of the spherical-harmonic tensor $\mathcal{Y}_{j m}^{\varrho}$ is proportional to a spin-weighted spherical harmonic ${ }_{s} Y_{j m}$. See Appendix A for a review of spin weight and spin-weighted harmonics. The key result of the calculation that follows is

$$
\left(\mathcal{Y}_{j m}^{\varrho}\right)_{+^{q_{+}-q_{-} r^{q_{r}}}}=\mathcal{Y}_{j m}^{\varrho} \cdot\left(\boldsymbol{e}_{+}^{\odot q_{+}} \odot \boldsymbol{e}_{-}^{\odot q_{-}} \odot \boldsymbol{e}_{r}^{\odot q_{r}}\right)={ }_{s} N_{j}^{\varrho \widehat{q}_{ \pm}}{ }_{s} Y_{j m},
$$

where it is understood that $\varrho=q_{+}+q_{-}+q_{r}, s=q_{+}-q_{-}$, and $\widehat{q}_{ \pm}=\min \left(q_{+}, q_{-}\right)$. In practice, the constraints can be handled by taking $q_{r}=\varrho-|s|, \varrho-|s|-2, \varrho-|s|-4, \ldots \geqslant$ 0 , with the remaining powers set to $q_{ \pm}=\frac{1}{2}\left(\varrho \pm s-q_{r}\right)$. The normalization constants ${ }_{s} N_{j}^{\varrho \widehat{q}_{ \pm}}$are zero unless $\varrho-j=$ even $\geqslant 0$, and the nonzero values are given by

$$
\begin{aligned}
{ }_{s} N_{j}^{\varrho \widehat{q}_{ \pm}}= & (-\operatorname{sgn} s)^{s} \frac{(\varrho-|s|) !}{(-2)^{\widehat{q}_{ \pm}}} \sqrt{\frac{4 \pi(j+|s|) !}{2^{|s|}(j-|s|) ! \varrho !(\varrho+j+1) ! !(\varrho-j) ! !}} \\
& \times{ }_{3} F_{2}\left(-\widehat{q}_{ \pm},-\frac{\varrho-j}{2},-\frac{\varrho+j+1}{2} ;-\frac{\varrho-|s|}{2},-\frac{\varrho-|s|-1}{2} ; 1\right),
\end{aligned}
$$

where ${ }_{3} F_{2}$ is a generalized hypergeometric function. The special case in Eq. (33) corresponds to $q_{+}=q_{-}=0$, and Eq. (18) is further restricted to $\varrho=j$. Note that some pow- ers $\left\{q_{+}, q_{-}, q_{r}\right\}$ obeying the above restrictions give vanishing ${ }_{s} N_{j}^{\varrho \widehat{q} \pm}$ constants, implying that the corresponding components of $\mathcal{Y}_{j m}^{\varrho}$ are zero. 
Equation (48) provides an alternative method for calculating the spin-weighted spherical harmonics. For example, we can relate the spin-weighted harmonics ${ }_{s} Y_{j m}$ to the trace-free rank- $j$ spherical-harmonic tensors $\mathcal{Y}_{j m}$ by setting $\varrho=j$. We can also take $q_{+}=0$ or $q_{-}=0$, which leads to the comparatively simple relation

${ }_{s} Y_{j m}=(-\operatorname{sgn} s)^{s} \sqrt{\frac{2^{|s|} j !(2 j+1) ! !}{4 \pi(j+s) !(j-s) !}} \mathcal{Y}_{j m} \cdot\left(\boldsymbol{e}_{ \pm}^{\odot|s|} \odot \boldsymbol{e}_{r}^{\odot(j-|s|)}\right)$,

where the sign on the $\boldsymbol{e}_{ \pm}$basis vector is the sign of $s$. This generalizes Eq. (18) to nonzero spin weight.

The derivation of Eqs. (48) and (49) starts by using spinweight ladder operators $J_{ \pm}=\boldsymbol{e}_{ \pm} \cdot \boldsymbol{J}=\frac{1}{\sqrt{2}}\left( \pm \partial_{\theta}+i \csc \theta \partial_{\phi}-\right.$ $s \cot \theta$ ) to raise and lower the spin weight of Eq. (33). With the conventional normalization, the spin-weighted harmonics are related through [9]

$$
J_{ \pm s} Y_{j m}=-\sqrt{\frac{1}{2}(j \pm s+1)(j \mp s)}{ }_{s \pm 1} Y_{j m} .
$$

Applying $J_{ \pm}$to the right-hand side of Eq. (33), we find that $J_{ \pm}$ converts one $\boldsymbol{n}=\boldsymbol{e}_{r}$ to $\boldsymbol{e}_{ \pm}$. This raises $q_{ \pm}$by 1 and lowers $q_{r}$ by 1 , incrementing the spin weight $s=q_{+}-q_{-}$but leaving $\varrho=q_{+}+q_{-}+q_{r}$ unchanged. Repeated application of ladder operators produces an expression of the form of Eq. (48) for the special case in which either $q_{+}$or $q_{-}$is zero, yielding

$$
\begin{aligned}
{ }_{s} N_{j}^{\varrho 0}= & (-\operatorname{sgn} s)^{s}(\varrho-|s|) ! \\
& \times \sqrt{\frac{4 \pi(j+|s|) !}{2^{|s|} \varrho !(j-|s|) !(\varrho+j+1) ! !(\varrho-j) ! !}} .
\end{aligned}
$$

For cases in which $q_{+}$and $q_{-}$are both nonzero, we use the completeness relation $g=2 \boldsymbol{e}_{+} \odot \boldsymbol{e}_{-}+\boldsymbol{e}_{r} \odot \boldsymbol{e}_{r}$ to write Eq. (48) as

$$
\begin{aligned}
{ }_{s} N_{j}^{\varrho \widehat{q}_{ \pm}}{ }_{s} Y_{j m} & =\mathcal{Y}_{j m}^{\varrho} \cdot\left(\boldsymbol{e}_{ \pm}^{\odot|s|} \odot \boldsymbol{e}_{r}^{\odot \varrho-|s|-2 \widehat{q}_{ \pm}} \odot \boldsymbol{e}_{+}^{\odot \widehat{q}_{ \pm}} \odot \boldsymbol{e}_{-}^{\odot \widehat{q}_{ \pm}}\right) \\
& =\left(\frac{1}{2}\right)^{\widehat{q}_{ \pm}} \mathcal{Y}_{j m}^{\varrho} \cdot\left[\boldsymbol{e}_{ \pm}^{\odot|s|} \odot \boldsymbol{e}_{r}^{\odot \varrho-|s|-2 \widehat{q}_{ \pm}} \odot\left(g-\boldsymbol{e}_{r} \odot \boldsymbol{e}_{r}\right)^{\odot \widehat{q}_{ \pm}}\right] \\
& =\left(-\frac{1}{2}\right)^{\widehat{q}_{ \pm}} \sum_{l}\left(\begin{array}{c}
\widehat{q}_{ \pm} \\
l
\end{array}\right)(-1)^{l} \mathcal{Y}_{j m}^{\varrho} \cdot\left(\boldsymbol{e}_{ \pm}^{\odot|s|} \odot \boldsymbol{e}_{r}^{\odot \varrho-|s|-2 l} \odot g^{\odot l}\right),
\end{aligned}
$$

where $\left(\begin{array}{l}p \\ q\end{array}\right)$ are binomial coefficients, and the index on the $\boldsymbol{e}_{ \pm}$basis vector matches the sign of $s$. The sum is limited to $0 \leqslant l \leqslant$ $\min \left(\widehat{q}_{ \pm}, \frac{1}{2}(\varrho-j)\right)$, where the limit $\frac{1}{2}(\varrho-j)$ is due to the tracelessness of $\mathcal{Y}_{j m}^{j}$. Using trace identity (42) and Eq. (48), we can write

$$
\begin{aligned}
{ }_{s} N_{j}^{\varrho \widehat{q}_{ \pm}}{ }_{s} Y_{j m} & =\left(-\frac{1}{2}\right)^{\widehat{q}_{ \pm}} \sum_{l}\left(\begin{array}{c}
\widehat{q}_{ \pm} \\
l
\end{array}\right)(-1)^{l} \sqrt{\frac{(\varrho-2 l) !(\varrho+j+1) ! !(\varrho-j) ! !}{\varrho !(\varrho+j+1-2 l) ! !(\varrho-j-2 l) ! !}} \mathcal{Y}_{j m}^{\varrho-2 l} \cdot\left(\boldsymbol{e}_{ \pm}^{\odot|s|} \odot \boldsymbol{e}_{r}^{\odot \varrho-|s|-2 l}\right) \\
& =\left(-\frac{1}{2}\right)^{\widehat{q_{ \pm}}} \sum_{l}\left(\begin{array}{c}
\widehat{q}_{ \pm} \\
l
\end{array}\right)(-1)^{l} \sqrt{\frac{(\varrho-2 l) !(\varrho+j+1) ! !(\varrho-j) ! !}{\varrho !(\varrho+j+1-2 l) ! !(\varrho-j-2 l) ! !}} N_{j}^{(\varrho-2 l) 0}{ }_{s} Y_{j m},
\end{aligned}
$$

which leads to

$$
{ }_{s} N_{j}^{\varrho \widehat{q}_{ \pm}}=(-\operatorname{sgn} s)^{s}\left(-\frac{1}{2}\right)^{\widehat{q_{ \pm}}} \sqrt{\frac{4 \pi(j+|s|) !(\varrho+j+1) ! !(\varrho-j) ! !}{2^{|s|} \varrho !(j-|s|) !}} \sum_{l}\left(\begin{array}{c}
\widehat{q}_{ \pm} \\
l
\end{array}\right) \frac{(-1)^{l}(\varrho-|s|-2 l) !}{(\varrho+j+1-2 l) ! !(\varrho-j-2 l) ! !} .
$$

Manipulating the factorials, one can show that the sum in this expression is equivalent to

$$
\frac{(\varrho-|s|) !}{(\varrho-j) ! !(\varrho+j+1) ! !}{ }_{3} F_{2}\left(-\widehat{q}_{ \pm},-\frac{\varrho-j}{2},-\frac{\varrho+j+1}{2} ;-\frac{\varrho-|s|}{2},-\frac{\varrho-|s|-1}{2} ; 1\right),
$$

which implies Eq. (49).

\section{ILLUSTRATIVE EXAMPLES}

The formalism developed in this work can be used to perform a full trace and angular-momentum decomposition of any tensor or tensor-valued function in three dimensions. As a simple example, consider the scalar function of the position vector $\boldsymbol{r}=x \boldsymbol{e}_{x}+y \boldsymbol{e}_{y}+z \boldsymbol{e}_{z}=r \boldsymbol{n}$ given by

$$
\begin{aligned}
f(\boldsymbol{r}) & =x^{2}+2 y z \\
& =r^{2}\left(\frac{1}{2} n_{\uparrow}^{2}+\frac{1}{2} n_{\downarrow}^{2}+n_{\uparrow} n_{\downarrow}-i \sqrt{2} n_{\uparrow} n_{z}+i \sqrt{2} n_{\downarrow} n_{z}\right) .
\end{aligned}
$$


We can write this as

$$
f(\boldsymbol{r})=r^{2} T^{a b} n_{a} n_{b},
$$

where the tensor $T$ can be taken as symmetric with nonzero Cartesian components $T^{x x}=T^{y z}=T^{z y}=1$. In the $J_{z}$ basis, the nonzero components are $T^{\uparrow \uparrow}=T^{\downarrow \downarrow}=T^{\uparrow \downarrow}=\frac{1}{2}$ and $T^{\downarrow z}=-T^{\uparrow z}=i / \sqrt{2}$. The spherical-harmonic expansion $f=$ $\sum_{j m} f_{j m} Y_{j m}(\boldsymbol{n})$ can be found by first expanding $T$ in the basis of rank-2 spherical-harmonic tensors: $T=\sum_{j m} T_{j m} \mathcal{Y}_{j m}^{2}$. The spherical components are the projections $T_{j m}=\mathcal{Y}_{j m}^{2 *} \cdot T$, which can be calculated using Eq. (38). The result is

$$
T_{2( \pm 2)}=\frac{1}{2}, \quad T_{2( \pm 1)}=i, \quad T_{20}=-\frac{1}{\sqrt{6}}, \quad T_{00}=\frac{1}{\sqrt{3}} .
$$

Note that the $j=2$ components give the traceless part of $T$, while $j=0$ is the trace component. Using Eq. (33) or Eq. (48), we can construct the spherical-harmonic expansion of $f$ :

$$
f(\boldsymbol{r})=\sum_{j m} r^{2} T_{j m} \mathcal{Y}_{j m}^{2} \cdot \boldsymbol{n}^{\odot 2}=\sum_{j m} r^{2} T_{j m 0} N_{j}^{20} Y_{j m}(\boldsymbol{n}) .
$$

So the spherical-harmonic coefficients for the function $f$ are

$$
f_{j m}=r^{2} T_{j m 0} N_{j}^{20}=r^{2} \sqrt{\frac{8 \pi}{(j+3) ! !(2-j) ! !}} T_{j m} .
$$

Note that these techniques allow for the algebraic construction of the spherical-harmonic expansion, providing an alternative to the standard method, where the coefficients are calculated through the solid-angle integrals $f_{j m}=\int Y_{j m}^{*} f \sin \theta d \theta d \phi$.

The scalar $f$ and the tensor $T$ in the above example both contain components with total angular momentum $j=0$ and 2. While $f$ has orbital angular momentum and $T$ has spin angular momentum, there is a third object involving $T$ that incorporates both spin and orbital angular momentum. The vector $\boldsymbol{V}(\boldsymbol{r})=T \cdot \boldsymbol{r}$ is a spin-1 function with orbital angular momentum. Its helicity-basis components are spin-weighted functions and can be expanded in spin-weighted spherical harmonics. The radial component $V_{r}=\boldsymbol{e}_{r} \cdot \boldsymbol{V}$ can be expanded in the usual $s=0$ spherical harmonics, while the $V_{ \pm}=\boldsymbol{e}_{ \pm} \cdot \boldsymbol{V}$ components are expanded in $s= \pm 1$ harmonics. The result is

$$
\begin{aligned}
V_{r} & =r \sum_{j m} T_{j m} \mathcal{Y}_{j m}^{2} \cdot\left(\boldsymbol{e}_{r} \odot \boldsymbol{e}_{r}\right)=r \sum_{j m} T_{j m 0} N_{j}^{20}{ }_{0} Y_{j m} \\
& =r \sum_{j m} \sqrt{\frac{8 \pi}{(j+3) ! !(2-j) ! !}} T_{j m 0} Y_{j m}, \\
V_{ \pm} & =r \sum_{j m} T_{j m} \mathcal{Y}_{j m}^{2} \cdot\left(\boldsymbol{e}_{ \pm} \odot \boldsymbol{e}_{r}\right)=r \sum_{m} T_{2 m \pm 1} N_{2}^{20}{ }_{ \pm 1} Y_{2 m} \\
& =\mp r \sum_{m} \sqrt{\frac{2 \pi}{5}} T_{2 m \pm 1} Y_{2 m} .
\end{aligned}
$$

Note that since spin weight is limited by $|s| \leqslant j, V_{ \pm}$only include $j=2$ components.

While the tensor $T$ is constant, its helicity-basis components are not. They can also be expanded in spin-weighted spherical harmonics:

$$
\begin{aligned}
& T_{r r}=\sum_{j m} T_{j m} \mathcal{Y}_{j m}^{2} \cdot\left(\boldsymbol{e}_{r} \odot \boldsymbol{e}_{r}\right)=\sum_{j m} T_{j m 0} N_{j}^{20}{ }_{0} Y_{j m} \\
& =\sum_{j m} \sqrt{\frac{8 \pi}{(j+3) ! !(2-j) ! !}} T_{j m 0} Y_{j m}, \\
& T_{+-}=\sum_{j m} T_{j m} \mathcal{Y}_{j m}^{2} \cdot\left(\boldsymbol{e}_{+} \odot \boldsymbol{e}_{-}\right)=\sum_{j m} T_{j m 0} N_{j}^{21}{ }_{0} Y_{j m} \\
& =\sum_{j m} \sqrt{\frac{\pi}{2(j+3) ! !(2-j) ! !}}\left(4-j-j^{2}\right) T_{j m}{ }_{0} Y_{j m}, \\
& T_{r \pm}=\sum_{j m} T_{j m} \mathcal{Y}_{j m}^{2} \cdot\left(\boldsymbol{e}_{r} \odot \boldsymbol{e}_{ \pm}\right)=\sum_{m} T_{2 m \pm 1} N_{2}^{20}{ }_{ \pm 1} Y_{2 m} \\
& =\mp \sum_{m} \sqrt{\frac{2 \pi}{5}} T_{2 m \pm 1} Y_{2 m}, \\
& T_{ \pm \pm}=\sum_{j m} T_{j m} \mathcal{Y}_{j m}^{2} \cdot\left(\boldsymbol{e}_{ \pm} \odot \boldsymbol{e}_{ \pm}\right)=\sum_{m} T_{m \pm 2} N_{2}^{20}{ }_{ \pm 2} Y_{2 m} \\
& =\sum_{m} \sqrt{\frac{4 \pi}{5}} T_{2 m \pm 2} Y_{2 m}
\end{aligned}
$$

Again, only $j=2$ contributes to the $s= \pm 1$ and \pm 2 components.

Notice that any constant symmetric tensor $T$ generates a set of functions with spins ranging from zero to its rank. The coefficients in the spherical-harmonic expansions of all these functions are related and differ by ${ }_{s} N_{j}^{\varrho \widehat{q}_{ \pm}}$factors. Also notice that we can construct the tensor $T$ given the sphericalharmonic expansions for any one of the functions in the set. For example, suppose we were given a scalar function $f(\boldsymbol{r})=\sum_{j m} r^{j} f_{j m} Y_{j m}(\boldsymbol{n})$ with known coefficients $f_{j m}$. Using Eq. (18) or Eq. (48), we can write $f$ in terms of the traceless spherical-harmonics tensors:

$$
f(\boldsymbol{r})=\sum_{j m} \frac{f_{j m}}{{ }_{0} N_{j}^{j 0}} \mathcal{Y}_{j m} \cdot \boldsymbol{r}^{\odot j}
$$

This function is the scalar in the set of tensor-valued functions generated by the constant tensor $T=\sum_{j m} \frac{f_{j m}}{{ }_{0} N_{j}^{j 0}} \mathcal{Y}_{j m}$.

The tensor decomposition of a function can also be used to quickly calculate derivatives of a function. For example, the gradient of the scalar function $f$ is the vector

$$
\nabla f=\sum_{j m} \frac{j f_{j m}}{{ }_{0} N_{j}^{j 0}} \mathcal{Y}_{j m} \cdot \boldsymbol{r}^{\odot(j-1)} .
$$

The helicity-basis components of the gradient have the spherical-harmonic expansions

$$
\begin{aligned}
& \boldsymbol{e}_{r} \cdot \nabla f=\sum_{j m} r^{j-1} j f_{j m} Y_{j m}(\boldsymbol{n}), \\
& \boldsymbol{e}_{ \pm} \cdot \nabla f=\sum_{j m} r^{j-1} j f_{j m} \frac{ \pm 1}{{ }_{0} N_{j}^{j 0}} N_{j}^{j 0} Y_{j m}(\boldsymbol{n}) .
\end{aligned}
$$

Vector-calculus operations like this can also be formulated in terms of the spin-weight ladder operators $J_{ \pm}$[9]. 
Finally, we note that while the methods developed here relate the spherical harmonics to symmetric tensors, they can be applied to other tensors. Any tensor can be split into symmetric tensors with the aid of Young symmetrizers, which are reviewed in Appendix B. As an example, consider an arbitrary rank-3 tensor $T$. A Young decomposition of the tensor reveals that it can be written as

$$
T=T_{S}+T_{A}+T_{1}+T_{2},
$$

where $T_{S}^{a b c}=\frac{1}{6} T^{(a b c)}$ is totally symmetric, and $T_{A}^{a b c}=$ $\frac{1}{6} T^{[a b c]}$ is totally antisymmetric. There are two mixedsymmetry pieces, $\quad T_{1}^{a b c}=\frac{1}{3}\left(T^{a b c}+T^{c b a}-T^{b a c}-T^{c a b}\right)$, which is antisymmetric in the first two indices, and $T_{2}^{a b c}=\frac{1}{3}\left(T^{a b c}+T^{b a c}-T^{c b a}-T^{b c a}\right)$, which is antisymmetric under interchange of the first index and last index. The symmetric part $T_{S}$ contains ten independent components, the antisymmetric $T_{A}$ has one independent component, and the mixed-symmetry parts have eight independent components each.

The formalism can be immediately applied to the symmetric part $T_{S}$. The antisymmetric part can be written as $T_{A}^{a b c}=$ $\frac{1}{3 !} \epsilon^{a b c} f$, where $\epsilon^{a b c}$ is the antisymmetric Levi-Civita tensor, and $f=\epsilon_{a b c}\left(T_{A}\right)^{a b c}$ is a scalar. The mixed symmetry pieces can be written as

$$
\begin{aligned}
& \left(T_{1}\right)^{a b c}=\epsilon_{d}^{a b}\left(t_{1}\right)^{c d}+\left(v_{1}\right)^{[a} g^{b] c}, \\
& \left(T_{2}\right)^{a b c}=\epsilon_{d}^{a c}\left(t_{2}\right)^{b d}+\left(v_{2}\right)^{[a} g^{c] b},
\end{aligned}
$$

where $t_{1}$ and $t_{2}$ are symmetric and traceless. This shows that any rank-3 tensor $T$ can be split into a rank-3 symmetric tensor, two traceless rank-2 symmetric tensors, two vectors, and a scalar. All of these symmetric tensors can be expanded in spherical harmonics using the above techniques.

\section{APPLICATION}

We now turn to a physics application. We use the sphericalharmonic tensors to calculate the leading-order effects of violations of Lorentz invariance in the gravitational potential $U$, providing an alternative to the approach currently found in the literature [10]. For experimental tests of Lorentz violation in Newtonian gravity, see Ref. [11].

Recent decades have seen renewed interest in challenging Lorentz invariance. These efforts are motivated, in part, by the observation that Lorentz invariance may be broken in theories of quantum gravity [12]. They were also spurred by the development of the standard-model extension (SME), a theoretical framework providing a general description of all realistic Lorentz violation in particles and in gravity [13]. The SME has served as the theoretical foundation for hundreds of searches for Lorentz violation [14].

The linearized limit of the gauge-invariant gravitational sector of the SME is given by the Lagrange density [15]

$$
\begin{aligned}
\mathcal{L}= & \frac{1}{4} \epsilon^{\mu \rho \alpha \kappa} \epsilon^{\nu \sigma \beta \lambda} \eta_{\kappa \lambda} h_{\mu \nu} \partial_{\alpha} \partial_{\beta} h_{\rho \sigma} \\
& +\sum_{d} \frac{1}{4} h_{\mu \nu} \mathcal{K}^{(d) \mu \nu \rho \sigma \alpha_{1} \cdots \alpha_{d-2}} \partial_{\alpha_{1}} \cdots \partial_{\alpha_{d-2}} h_{\rho \sigma},
\end{aligned}
$$

where $h_{\mu \nu}$ is the deviation of the spacetime metric from the constant Minkowski metric $\eta_{\mu \nu}$. The first term in Eq. (69) is the usual linearized Einstein-Hilbert Lagrangian, which describes conventional gravity in the weak-field limit. The remaining parts include all possible Lorentz-violating terms that are quadratic in $h_{\mu \nu}$, translationally invariant, and invariant under the usual gauge transformation, $h_{\mu \nu} \rightarrow h_{\mu \nu}+\partial_{(\mu} \xi_{v)}$. The Lorentz violation is controlled by the $\mathcal{K}^{(d)}$ spacetimetensor coefficients. A Young decomposition splits the tensors into three classes of coefficients for Lorentz violation, $s^{(d)}$, $q^{(d)}$, and $k^{(d)}$, with the symmetries given in Table 1 of Ref. [15]. The $s^{(d)}$ coefficients are nonzero for even $d \geqslant 4$, the $q^{(d)}$ are nonzero for odd $d \geqslant 5$, and the $k^{(d)}$ are nonzero for even $d \geqslant 6$.

The modified equations of motion arising from Eq. (69) provide a test theory for studies of Lorentz violation in gravitational waves $[15,16]$ and in Newtonian gravity. Assuming a static mass distribution $\rho(\boldsymbol{x})$, it can be shown that the Lorentzviolating contributions to the Newtonian potential are given by [10]

$$
\delta U=\frac{1}{4} \sum_{d} \mathcal{K}^{(d) \mu \mu v v a_{1} a_{2} \cdots a_{d-2}} \partial_{a_{1}} \partial_{a_{2}} \cdots \partial_{a_{d-2}} \chi,
$$

where $\partial_{a}=\partial / \partial x^{a}$ are spatial derivatives, and $d$ is now restricted to even values. The "superpotential" $\chi$ is defined as

$$
\chi(\boldsymbol{x})=-G_{N} \int d^{3} x^{\prime}\left|\boldsymbol{x}-\boldsymbol{x}^{\prime}\right| \rho\left(\boldsymbol{x}^{\prime}\right),
$$

where $G_{N}$ is Newton's constant. Both the conventional potential $U=-\frac{1}{2} \nabla^{2} \chi=G_{N} \int d^{3} x^{\prime} \rho\left(\boldsymbol{x}^{\prime}\right) /\left|\boldsymbol{x}-\boldsymbol{x}^{\prime}\right|$ and the Lorentz-violating potential $\delta U$ can by found by taking derivatives of $\chi$.

Using the above equations, one can calculate the effects of Lorentz violation on gravity from a mass distribution $\rho$, and experimental constraints can be placed on the $\mathcal{K}^{(d) \mu \mu \nu v a_{1} a_{2} \cdots a_{d-2}}$ coefficient combinations. This is commonly done by searching for variations in an experimental signal while rotating the apparatus. In an inertial frame, these rotations change the mass distribution $\rho$ and the superpotential $\chi$ but not the $\mathcal{K}^{(d)}$ coefficients. Alternatively, we can work in a noninertial apparatus-fixed frame in which $\chi$ is constant but the $\mathcal{K}^{(d)}$ coefficients rotate. In either case, the potential $\delta U$ varies with these rotations. The frames commonly used in these types of experiments and the rotations relating them are discussed in Appendix A.

The significant role played by rotations in tests of Lorentz invariance prompts an angular-momentum decomposition of Eq. (70). In Ref. [10], this is done by switching to momentum space, which results in the replacement $\partial_{a} \rightarrow i p_{a}$. The $p$-space potential $\delta U(\boldsymbol{p})$ is then expanded in spherical harmonics. Using a Fourier transform to switch back to position space, one finds that the Lorentz-violating potential takes the from

$$
\delta U(\boldsymbol{x})=G_{N} \sum_{d j m} k_{j m}^{\mathrm{N}(d)} \int d^{3} x^{\prime} \frac{Y_{j m}(\hat{\boldsymbol{r}}) \rho\left(\boldsymbol{x}^{\prime}\right)}{|\boldsymbol{r}|^{d-3}},
$$

where the sum is restricted to even $j$ and $d=j+2, j+4$. The vector $\boldsymbol{r}=\boldsymbol{x}-\boldsymbol{x}^{\prime}$ is the position relative to the source, and $\hat{\boldsymbol{r}}=\boldsymbol{r} /|\boldsymbol{r}|$ is the direction. The spherical Newton coefficients 
for Lorentz violation $k_{j m}^{\mathrm{N}(d)}$ are the linear combinations of components of $\mathcal{K}^{(d)}$ that affect Newtonian gravity at leading order. The tools developed in this work provide for an alternative derivation and can be used to find the relationship between the spherical Newton coefficients and the coefficient tensors that appear in the Lagrange density.

We first expand in spherical-harmonic tensors,

$$
\mathcal{K}^{(d) \mu \mu \nu v a_{1} a_{2} \cdots a_{d-2}}=\sum_{j m} \mathcal{K}_{j m}^{(d)}\left(\mathcal{Y}_{j m}^{d-2}\right)^{a_{1} a_{2} \cdots a_{d-2}},
$$

where

$$
\mathcal{K}_{j m}^{(d)}=\mathcal{K}^{(d) \mu \mu \nu v a_{1} a_{2} \cdots a_{d-2}}\left(\mathcal{Y}_{j m}^{(d-2) *}\right)_{a_{1} a_{2} \cdots a_{d-2}} .
$$

The Lorentz-violating potential can then be written

$$
\begin{aligned}
\delta U= & \frac{1}{4} \sum_{d j m} \sqrt{\frac{(d-2) !(2 j+1) ! !}{j !(d+j-1) ! !(d-j-2) ! !}} \\
& \times \mathcal{K}_{j m}^{(d)}\left(\mathcal{Y}_{j m}\right)^{a_{1} a_{2} \cdots a_{j}} \nabla^{d-j-2} \partial_{a_{1}} \partial_{a_{2}} \cdots \partial_{a_{j}} \chi,
\end{aligned}
$$

where $\nabla^{2}=\partial_{a} \partial^{a}$ is the Laplacian. Since $\nabla^{4} \chi=-2 \nabla^{2} U=0$ outside the mass distribution, only $d=j+2$ and $d=j+$ 4 contribute, giving the restriction on $d$ described above. Next consider spatial-derivative operators $\partial_{a}$ acting on the $\left|\boldsymbol{x}-\boldsymbol{x}^{\prime}\right|=|\boldsymbol{r}|$ appearing in the superpotential (71). Two derivatives give $\partial_{a_{1}} \partial_{a_{2}}|\boldsymbol{r}|=g_{a_{1} a_{2}} /|\boldsymbol{r}|-r_{a_{1}} r_{a_{2}} /|\boldsymbol{r}|^{3}$. The term involving the metric will not contribute since $\mathcal{Y}_{j m}$ is traceless. Similar irrelevant terms result when taking higher derivatives. After a short calculation, this results in

$$
\begin{aligned}
\delta U= & \frac{1}{4} G_{N} \sum_{d j m} \xi_{j}^{d}(2 j-3) ! ! \sqrt{\frac{(d-2) !(2 j+1) ! !}{j !(d+j-1) ! !(d-j-2) ! !}} \\
& \times \mathcal{K}_{j m}^{(d)}\left(\mathcal{Y}_{j m}\right)^{a_{1} a_{2} \cdots a_{j}} \int d^{3} x^{\prime} \frac{r_{a_{1}} \cdots r_{a_{j}}}{|\boldsymbol{r}|^{d+j-3}} \rho\left(\boldsymbol{x}^{\prime}\right),
\end{aligned}
$$

where $\xi_{j}^{d}=1$ when $d=j+2, \xi_{j}^{d}=2-4 j$ when $d=j+4$, and $\xi_{j}^{d}=0$ otherwise. Using Eq. (18), the Lorentz-violating potential reduces to Eq. (72), where

$$
k_{j m}^{\mathrm{N}(d)}=\frac{1}{4} \xi_{j}^{d}(2 j-3) ! ! \sqrt{\frac{4 \pi(d-2) !}{(d+j-1) ! !(d-j-2) ! !}} \mathcal{K}_{j m}^{(d)} .
$$

Combined with Eq. (74), this gives the relationship between the Newton coefficients and the coefficient tensors in the Lagrange density of the theory.

Finding $\delta U$ using Eq. (72) requires calculating a different integral for each $k_{j m}^{\mathrm{N}(d)}$ coefficient. This "many-integrals" approach may be computationally expensive. The sphericalharmonic tensors lead to a "many-derivatives" alternative,

$$
\delta U=\frac{1}{4} \sum_{d j m} \mathcal{K}_{j m}^{(d)}\left(\mathcal{Y}_{j m}^{d-2}\right)^{a_{1} a_{2} \cdots a_{d-2}} \partial_{a_{1}} \partial_{a_{2}} \cdots \partial_{a_{d-2}} \chi,
$$

which may be easier to compute. Only a single integral is required in order to calculate the superpotential, and the effects of Lorentz violation are then found by taking its derivatives.

\section{SUMMARY}

In this work, we construct an orthonormal set of symmetric spherical-harmonic tensors. The most general versions are the rank- $\varrho$ tensors $\mathcal{Y}_{j m}^{\varrho}$ given in Eq. (31). Their connection to spherical harmonics $Y_{j m}$ is given in Eq. (33) and to spinweighted spherical harmonics ${ }_{s} Y_{j m}$ in Eq. (48). In the case in which the rank $\varrho=j$, the tensors $\mathcal{Y}_{j m}=\mathcal{Y}_{j m}^{j}$ are traceless and reduce to Eq. (19). Equation (18) gives the relation between the $\mathcal{Y}_{j m}$ tensors and the scalar $s=0$ spherical harmonics $Y_{j m}$. The $\mathcal{Y}_{j m}^{\varrho}$ are constant tensors with spin eigenvalues $J^{2}=$ $j(j+1)$ and $J_{z}=m$ and form an angular-momentum basis for symmetric rank- $\varrho$ tensors. Any constant rank- $\varrho$ tensor $T$ can be expanded in $\mathcal{Y}_{j m}^{\varrho}$, providing a full angular-momentum and trace decomposition.

Section III contains several examples illustrating how the $\mathcal{Y}_{j m}^{\varrho}$ can be used to expand tensors and tensor-valued functions in $\mathcal{Y}_{j m}^{\varrho}$ and how they are connected to spherical-harmonic expansions. An application of the formalism involving the study of violations of Lorentz invariance is discussed in Sec. IV. The leading-order effects of potential Lorentz violation in Newtonian gravity are formulated in terms of derivatives of a gravitational superpotential using the $\mathcal{Y}_{j m}$ tensors, providing a "many-derivatives" alternative to the "many-integrals" approach that is currently found in the literature.

\section{ACKNOWLEDGMENTS}

This work was supported in part by the William and Linda Frost Fund and by the U.S. National Science Foundation under Grant No. PHY-1819412.

\section{APPENDIX A: SPIN-WEIGHTED SPHERICAL HARMONICS}

Spin-weighted spherical harmonics are a form of tensor spherical harmonics and provide an angular-momentum decomposition for functions with both nonzero spin and orbital angular momentum. This Appendix briefly reviews spin weight and spin-weighted spherical harmonics. A more detailed discussion can be found in Refs. [6-9].

To understand spin weight, first consider the various sets of compatible angular-momentum operators. The physics literature typically focuses on the product-space basis, comprised of eigenfunctions of the operators $\left\{S^{2}, L^{2}, S_{z}, L_{z}\right\}$, and the total-angular-momentum basis, given by the eigenfunctions of $\left\{S^{2}, L^{2}, J^{2}, J_{z}\right\}$. However, a third set exists, $\left\{S^{2}, J^{2}, J_{z}, J_{r}\right\}$, where the helicity $J_{r}=\boldsymbol{n} \cdot \boldsymbol{J}=\boldsymbol{n} \cdot \boldsymbol{S}$ is the component of the total angular momentum or spin angular momentum along the $\boldsymbol{n}$ direction. The spin-weighted spherical harmonics ${ }_{s} Y_{j m}(\boldsymbol{n})$ are eigenfunctions of $J^{2}, J_{z}$, and $J_{r}$. By convention, the spin weight $s$ is defined so that it is the eigenvalue of $-J_{r}$, implying that spin weight is the opposite of helicity. It is limited by $-j \leqslant s \leqslant j$ since it is a component of the total angular momentum. The usual harmonics correspond to the $s=0$ case, $Y_{j m}={ }_{0} Y_{j m}$. More generally, the spin-weighted spherical harmonics ${ }_{s} Y_{j m}$ form an orthonormal basis for spin-weighted functions and provide for the angular-momentum expansion of higher-rank tensor functions. 
A function $f(\boldsymbol{n})$ is said to have spin-weight $s$ if it transforms according to $f \rightarrow e^{-i s \alpha} f$ under an active rotation about $\boldsymbol{n}$ by angle $\alpha$. These rotations are generated by the helicity operator $J_{r}=S_{r}$. This only depends on spin since orbital angular momentum $\boldsymbol{L}$ transforms the argument of a function, and $\boldsymbol{n}$ is invariant under these rotations. Spin $\boldsymbol{S}$ accounts for the directionality of an object. Spin-weighted functions with $s \neq 0$ change under rotations generated by $S_{r}$, implying they are necessarily directional or tensoral in nature. The reverse is also true. Tensors of nonzero rank have spin weight. More specifically, the components of a tensor in the helicity basis $\left\{\boldsymbol{e}_{r}, \boldsymbol{e}_{+}, \boldsymbol{e}_{-}\right\}$are spin-weighted functions [9].

The spin weight of a helicity-basis component of a tensor is determined by the number of + or - indices. Each lowered \pm index or raised $\mp$ index contributes \pm 1 to the spin weight. For example, a rank-3 tensor has six $s=1$ components$T_{+r r}, T_{r+r}, T_{r r+}, T_{++-}, T_{+-+}$, and $T_{-++}$- each of which can be expanded in the ${ }_{1} Y_{j m}$ spherical harmonics. In total, the helicity-basis components of $T$ give 27 spin-weighted functions: one for each of $s= \pm 3$, three for each of $s= \pm 2$, six for each of $s= \pm 1$, and seven with $s=0$. In general, the spin weight of the components of a rank- $\varrho$ tensor is limited by $-\varrho \leqslant s \leqslant \varrho$.

Many of the usual spherical-harmonic identities can be extended to the spin-weighted harmonics. The harmonics of equal spin weight are orthonormal,

$$
\int{ }_{s} Y_{j m}^{*} Y_{j^{\prime} m^{\prime}} \sin \theta d \theta d \phi=\delta_{j j^{\prime}} \delta_{m m^{\prime}},
$$

and satisfy the completeness relations

$$
\sum_{j m}{ }_{s} Y_{j m}^{*}(\theta, \phi){ }_{s} Y_{j m}\left(\theta^{\prime}, \phi^{\prime}\right)=\frac{\delta\left(\theta-\theta^{\prime}\right) \delta\left(\phi-\phi^{\prime}\right)}{\sin \theta} .
$$

Assuming a Condon-Shortley phase, they obey the complexconjugation rule

$$
{ }_{s} Y_{j m}^{*}=(-1)^{s+m}{ }_{-s} Y_{j(-m)} .
$$

They also obey the parity relation

$$
{ }_{s} Y_{j m}(-\boldsymbol{n})=(-1)^{j}{ }_{-s} Y_{j m}(\boldsymbol{n}) .
$$

The product of two harmonics is

$$
\begin{aligned}
& s_{1} Y_{j_{1} m_{1} s_{2}} Y_{j_{2} m_{2}} \\
& =\sum_{s_{3} j_{3} m_{3}} \sqrt{\frac{\left(2 j_{1}+1\right)\left(2 j_{2}+1\right)}{4 \pi\left(2 j_{3}+1\right)}}\left\langle j_{1} j_{2}\left(-s_{1}\right)\left(-s_{2}\right) \mid j_{3}\left(-s_{3}\right)\right\rangle \\
& \quad \times\left\langle j_{1} j_{2} m_{1} m_{2} \mid j_{3} m_{3}\right\rangle_{s_{3}} Y_{j_{3} m_{3}},
\end{aligned}
$$

where $\left\langle j_{1} j_{2} m_{1} m_{2} \mid j_{3} m_{3}\right\rangle$ are Clebsch-Gordan coefficients.

The spin-weighted harmonics are eigenfunctions of the square of the total angular momentum $J^{2}$, the $z$-component of the total angular momentum $J_{z}$, and the helicity $J_{r}$, with eigenvalues $J^{2}=j(j+1), J_{z}=m$, and $J_{r}=-s$. The spin-weighted harmonics transform relatively simply under rotations since they are generated by angular momentum $\vec{J}$. All three components of $\vec{J}$ commute with both $J^{2}$ and $J_{r}$, implying that quantum numbers $j$ and $s$ are invariant under rotations, and rotations only mix harmonics with different $m$ values, leaving $j-s$ subspaces invariant. The mixing is characterized by the Wigner matrices, defined through

$$
D_{m m^{\prime}}^{(j)}(\alpha, \beta, \gamma)=\int{ }_{s} Y_{j m}^{*} e^{-i \alpha J_{z}} e^{-i \beta J_{y}} e^{-i \gamma J_{z}}{ }_{s} Y_{j m^{\prime}} \sin \theta d \theta d \phi,
$$

where $\alpha, \beta$, and $\gamma$ are Euler angles. Note that Eq. (A6) assumes $|s| \leqslant j$, but is otherwise independent of the the spin weight $s$. The above employs a $z-y-z$ rotation convention, which is advantageous since the spherical harmonics are eigenfunctions for $z$ rotations, leading to simple phases for two of the Euler angles,

$$
D_{m m^{\prime}}^{(j)}(\alpha, \beta, \gamma)=e^{-i \alpha m} e^{-i \gamma m^{\prime}} d_{m m^{\prime}}^{(j)}(\beta),
$$

where $d_{m m^{\prime}}^{(j)}(\beta)=D_{m m^{\prime}}^{(j)}(0, \beta, 0)$ are the little Wigner matrices.

Operating on the components of a tensor, $e^{-i \alpha J_{z}} e^{-i \beta J_{y}} e^{-i \gamma J_{z}}$ rotates tensor components by $\gamma$ about the $z$ axis, then by $\beta$ about the $y$ axis, and finally by $\alpha$ about the $z$ axis. Interpreting this as a passive transformation, this corresponds to rotating the coordinate axes by $-\gamma$ about the $z$ axis, then $-\beta$ about the rotated $y$ axis, and then by $-\alpha$ about the new $z$ axis. The Cartesian components of the old frame $\{x, y, z\}$ and the rotated frame $\left\{x^{\prime}, y^{\prime}, z^{\prime}\right\}$ are related through

$$
\begin{aligned}
\left(\begin{array}{l}
x^{\prime} \\
y^{\prime} \\
z^{\prime}
\end{array}\right)= & \left(\begin{array}{ccc}
\cos \alpha & -\sin \alpha & 0 \\
\sin \alpha & \cos \alpha & 0 \\
0 & 0 & 1
\end{array}\right)\left(\begin{array}{ccc}
\cos \beta & 0 & \sin \beta \\
0 & 1 & 0 \\
-\sin \beta & 0 & \cos \beta
\end{array}\right) \\
& \times\left(\begin{array}{ccc}
\cos \gamma & -\sin \gamma & 0 \\
\sin \gamma & \cos \gamma & 0 \\
0 & 0 & 1
\end{array}\right)\left(\begin{array}{l}
x \\
y \\
z
\end{array}\right) .
\end{aligned}
$$

The basis vectors $\left\{\boldsymbol{e}_{x}, \boldsymbol{e}_{y}, \boldsymbol{e}_{z}\right\}$ and the rotated-basis vectors $\left\{\boldsymbol{e}_{x}^{\prime}, \boldsymbol{e}_{y}^{\prime}, \boldsymbol{e}_{z}^{\prime}\right\}$ also obey this relation.

We can transform the spherical-harmonic expansion of a function using the Wigner matrices. A spin-weighted function $f(\boldsymbol{n})=\sum_{j m} f_{j m s} Y_{j m}(\boldsymbol{n})$ rotates according to $f^{\prime}(\boldsymbol{n})=$ $\sum_{j m} f_{j m s}^{\prime} Y_{j m}(\boldsymbol{n})=e^{-i \alpha J_{z}} e^{-i \beta J_{y}} e^{-i \gamma J_{z}} f(\boldsymbol{n})$, giving rotated expansion coefficients

$$
f_{j m}^{\prime}=\sum_{m^{\prime}} D_{m m^{\prime}}^{(j)}(\alpha, \beta, \gamma) f_{j m^{\prime}}
$$

Consider, for example, rotations of a laboratory due to the daily rotation of the Earth. Standard reference frames appear in the literature to account for this rotation $[9,14]$. A nonrotating Sun-centered frame is defined so that the $\hat{Z}$ axis points along the Earth's rotation axis, and $\hat{\boldsymbol{X}}$ and $\hat{\boldsymbol{Y}}$ lie in the equatorial plane with right ascension $0^{\circ}$ and $90^{\circ}$, respectively. A rotating laboratory-fixed frame is defined with $\hat{z}$ pointing up and $\hat{\boldsymbol{x}}$ and $\hat{\boldsymbol{y}}$ horizontal with $\hat{\boldsymbol{x}}$ at an angle $\varphi$ measured east of south. The coordinates in these two frames are related through

$$
\begin{aligned}
\left(\begin{array}{l}
X \\
Y \\
Z
\end{array}\right)= & \left(\begin{array}{ccc}
\cos \alpha & -\sin \alpha & 0 \\
\sin \alpha & \cos \alpha & 0 \\
0 & 0 & 1
\end{array}\right)\left(\begin{array}{ccc}
\cos \chi & 0 & \sin \chi \\
0 & 1 & 0 \\
-\sin \chi & 0 & \cos \chi
\end{array}\right) \\
& \times\left(\begin{array}{ccc}
\cos \varphi & -\sin \varphi & 0 \\
\sin \varphi & \cos \varphi & 0 \\
0 & 0 & 1
\end{array}\right)\left(\begin{array}{l}
x \\
y \\
z
\end{array}\right)
\end{aligned}
$$


where $\chi$ is the colatitude of the laboratory, and $\alpha$ is the right ascension of the laboratory zenith. The spherical-expansion coefficients in the two frames are related through

$$
\begin{aligned}
f_{j m}^{\mathrm{Sun}} & =\sum_{m^{\prime}} D_{m m^{\prime}}^{(j)}(\alpha, \chi, \varphi) f_{j m^{\prime}}^{\mathrm{lab}}, \\
f_{j m}^{\mathrm{lab}} & =\sum_{m^{\prime}} D_{m m^{\prime}}^{(j)}(-\varphi,-\chi,-\alpha) f_{j m^{\prime}}^{\mathrm{Sun}} .
\end{aligned}
$$

Note that $\alpha$ increases at Earth's sidereal rate $\approx 2 \pi / 23 \mathrm{~h} 56 \mathrm{~min}$ due to the daily rotation of the Earth. It is common for experiments to be placed on horizontal turntables so that the angle $\varphi$ varies as well.

\section{APPENDIX B: YOUNG SYMMETRIZERS}

This Appendix provides a brief overview of Young symmetrizers and their use in the symmetry decomposition of tensors. For an in-depth treatment, see, e.g., Refs. [17,18].

The splitting of a rank-2 tensor $T^{a b}$ into its symmetric part $T_{\mathrm{S}}^{a b}=\frac{1}{2} T^{(a b)}=\frac{1}{2}\left(T^{a b}+T^{b a}\right)$ and its antisymmetric part $T_{\mathrm{A}}^{a b}=\frac{1}{2} T^{[a b]}=\frac{1}{2}\left(T^{a b}-T^{b a}\right)$ is a simple example of Young symmetrization. Each of these pieces transforms under a different irreducible representation of the general linear group $G L_{n}$, meaning they do not mix under linear transformations of the underlying $n$-dimensional vector space. Note that a trace decomposition provides additional reduction for orthogonal subgroups, such as rotations or Lorentz transformations. For example, the trace decomposition of a rank-2 tensor yields $T^{a b}=\bar{T}_{\mathrm{S}}^{a b}+T_{\mathrm{A}}^{a b}+\frac{1}{n} g^{a b} T_{\mathrm{tr}}$, where $T_{\mathrm{tr}}=T^{a b} g_{a b}$ is the trace, and $\bar{T}_{\mathrm{S}}^{a b}=T_{\mathrm{S}}^{a b}-\frac{1}{n} g^{a b} T_{\text {tr }}$ is the traceless symmetric part.

Tensors $T^{a_{1} \cdots a_{\varrho}}$ with larger rank $\varrho>2$ contain additional mixed-symmetry parts. These can be constructed using Young symmetry projections, each of which can be represented graphically as a Young tableau. The construction of a tableau starts by selecting indices from $T^{a_{1} \cdots a_{e}}$ in any order. Here we take them in the order they appear and start by drawing a single-box tableau $a_{1}$ for the first index. We then combine this with the next index to produce the two-box tableaus:

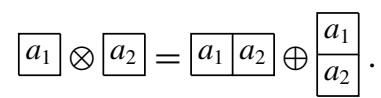

Each subsequent index creates a new row or extends an existing row. For example, the three-box tableaus are

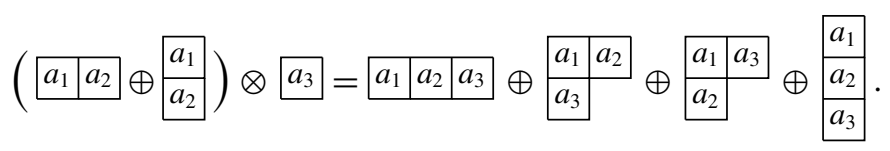

Adding another index gives

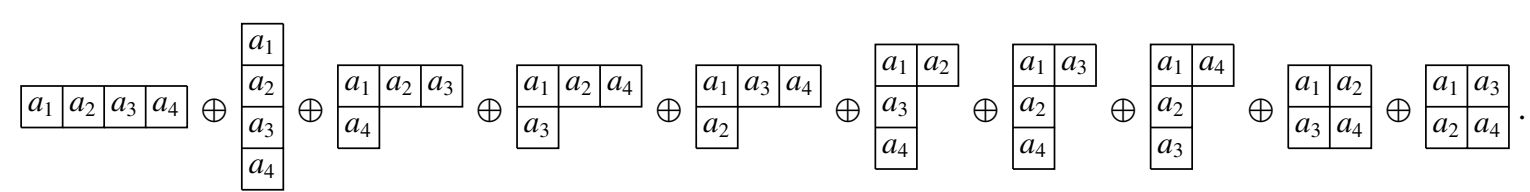

One continues in this fashion for all $\varrho$ indices. Note that this produces a smaller number of shapes known as Young diagrams, and that tableaus with the same diagram are related through permutations of indices.

Young symmetrizers are combinations of symmetrization operators $\mathcal{S}$ and antisymmetrization operators $\mathcal{A}$, defined so that $\mathcal{S}\left(a_{2} a_{3} a_{4}\right) T^{a_{1} a_{2} a_{3} a_{4} a_{5} \cdots}=T^{a_{1}\left(a_{2} a_{3} a_{4}\right) a_{5} \cdots}$ and $\mathcal{A}\left(a_{2} a_{3} a_{4}\right) T^{a_{1} a_{2} a_{3} a_{4} a_{5} \cdots}=T^{a_{1}\left[a_{2} a_{3} a_{4}\right] a_{5} \cdots}$, for example. The Young symmetrizer $\mathcal{P}$ associated with a given tableau is constructed by first symmetrizing on indices in each row and then antisymmetrizing on indices in each column. For example, the Young symmetrizers for the $\varrho=4$ tableaus are

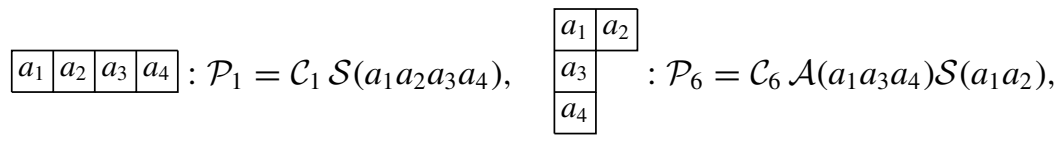

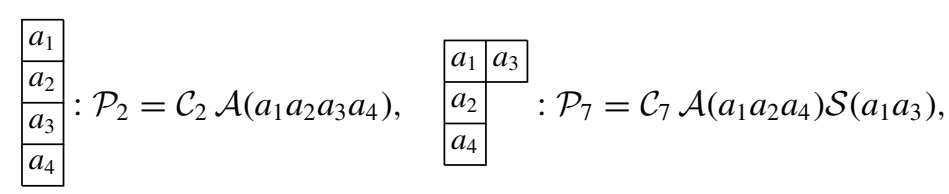

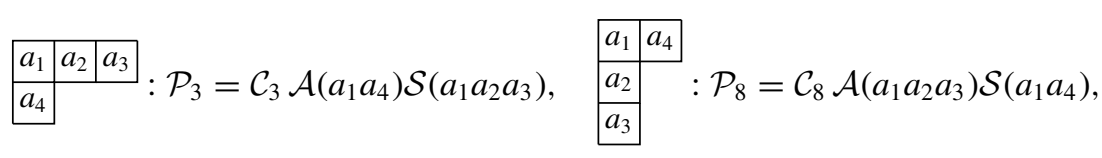

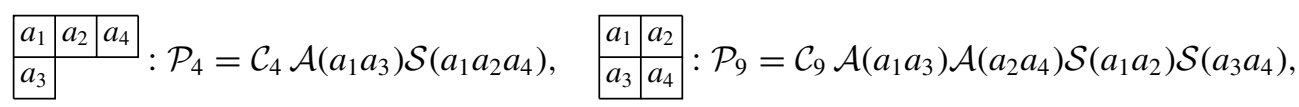

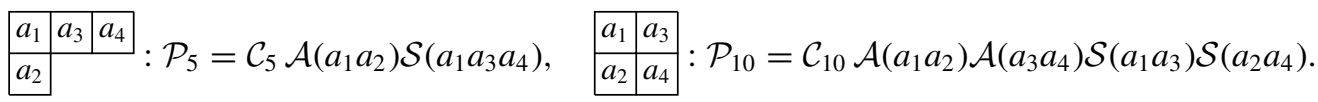




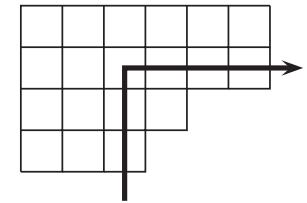

FIG. 1. Hook for row $r=2$ and column $c=3$ with hook length $h_{2,3}=6$.

The $\mathcal{C}_{\iota}$ constants are chosen so that the $\mathcal{P}_{\iota}$ form a complete set of orthogonal projection operators: $\sum_{l} \mathcal{P}_{\iota}=$ identity, $\mathcal{P}_{\iota} \mathcal{P}_{\iota^{\prime}}=$ $\mathcal{P}_{\iota} \delta_{\iota^{\prime}}$. The projections $T_{\iota}=\mathcal{P}_{\iota} T$ give parts of a tensor $T=$ $\sum_{l} T_{l}$ that transform under irreducible representations of $G L_{n}$. For example, $\mathcal{P}_{9}$ from above gives the tensor

$$
\begin{aligned}
T_{9}^{a_{1} a_{2} a_{3} a_{4}}= & \mathcal{C}_{9}\left(T^{a_{1} a_{2} a_{3} a_{4}}+T^{a_{2} a_{1} a_{3} a_{4}}+T^{a_{1} a_{2} a_{4} a_{3}}+T^{a_{2} a_{1} a_{4} a_{3}}\right. \\
& +T^{a_{3} a_{4} a_{1} a_{2}}+T^{a_{4} a_{3} a_{1} a_{2}}+T^{a_{3} a_{4} a_{2} a_{1}}+T^{a_{4} a_{3} a_{2} a_{1}} \\
& -T^{a_{3} a_{2} a_{1} a_{4}}-T^{a_{2} a_{3} a_{1} a_{4}}-T^{a_{3} a_{2} a_{4} a_{1}}-T^{a_{2} a_{3} a_{4} a_{1}} \\
& \left.-T^{a_{1} a_{4} a_{3} a_{2}}-T^{a_{4} a_{1} a_{3} a_{2}}-T^{a_{1} a_{4} a_{2} a_{3}}-T^{a_{4} a_{1} a_{2} a_{3}}\right) .
\end{aligned}
$$

Note $T_{1}$ and $T_{2}$ in our example are the fully symmetric and antisymmetric parts. The other parts are said to have mixed symmetry. Also note that the $T_{\iota}$ are antisymmetric in indices appearing in each column of the tableau. This implies that a $T_{\iota}$ vanishes if the number of rows in the tableau exceeds the dimension $n$ of the space. The $T_{\iota}$ are not symmetric in indices appearing in the rows. However, different conventions exist, including ones where $\mathcal{S}$ operations follow $\mathcal{A}$ operations, leaving the $T_{\iota}$ symmetric in row indices.

The mixed-symmetry $T_{\iota}$ can have complicated symmetries leading to more subtle features, which can be uncovered using symmetrizers. For example, the antisymmetrization of any three indices of $T_{9}^{a_{1} a_{2} a_{3} a_{4}}$ vanishes since

$$
\begin{aligned}
\mathcal{A}\left(a_{1} a_{2} a_{3}\right) \mathcal{P}_{9} & =\mathcal{C}_{9} \mathcal{A}\left(a_{1} a_{2} a_{3}\right) \mathcal{A}\left(a_{1} a_{2}\right) \mathcal{S}\left(a_{1} a_{3} a_{4}\right) \\
& =2 \mathcal{C}_{9} \mathcal{A}\left(a_{1} a_{2} a_{3}\right) \mathcal{S}\left(a_{1} a_{3} a_{4}\right)=0 .
\end{aligned}
$$

The same holds for any choice of three indices.

The $\mathcal{C}_{l}$ normalization constants and the number $\mathcal{N}_{l}$ of independent components for a given $T_{l}$ can be easily calculated using the hook lengths $h_{r, c}$ for the diagram [19]. A hook is a path through a Young diagram moving up through the bottom of the diagram to the box in row $r$ and column $c$ and then out of the diagram to the right. The hook length is the number boxes the hook passes through. An example is shown in Fig. 1. The $\mathcal{C}_{\iota}$ normalization constant is the reciprocal of the product of all the hook lengths for the diagram,

$$
\mathcal{C}_{\iota}=\prod_{r, c} \frac{1}{h_{r, c}}
$$

and the number of independent components in tensor $T_{\iota}$ is given by

$$
\mathcal{N}_{\iota}=\prod_{r, c} \frac{n+c-r}{h_{r, c}}
$$

where again $n$ is the dimension of the space. As an example, the $\mathcal{P}_{9}$ above has normalization constant $\mathcal{C}_{9}=1 / 12$, and the tensor $T_{9}$ has $\mathcal{N}_{9}=(n+1) n^{2}(n-1) / 12$ independent components. The independent components can be taken as those corresponding to a semistandard tableau, i.e., ones where the index values increase as one moves down a column, and do not decrease when moving to the right in a row. For example, taking $n=3$ and working in Cartesian coordinates $\{x, y, z\}$, the $\mathcal{N}_{9}=6$ semistandard tableaus for $\mathcal{P}_{9}$ are

\begin{tabular}{|l|l|}
\hline$a_{1}$ & $a_{2}$ \\
\hline$a_{3}$ & $a_{4}$ \\
\hline
\end{tabular}$=$\begin{tabular}{|l|l|}
\hline$x$ & $x$ \\
\hline$y$ & $y$ \\
\hline$y$ & $z$ \\
\hline$x$ & $x$ \\
\hline$z$ & $z$ \\
\hline$x$ & $x$ \\
\hline$y$ & $z$ \\
\hline
\end{tabular}, \begin{tabular}{|l|l|}
\hline$x$ & $y$ \\
\hline$z$ & $z$ \\
\hline$z$ & $z$ \\
\hline
\end{tabular}.

Using the symmetries of $T_{9}$, all of its components $T_{9}^{a_{1} a_{2} a_{3} a_{4}}$ can be written as linear combinations of $T_{9}^{x x y y}, T_{9}^{x x y z}, T_{9}^{x x z z}, T_{9}^{x y y z}$, $T_{9}^{x y z z}$, and $T_{9}^{y y z z}$.
[1] J. Applequist, J. Phys. A 22, 4303 (1989).

[2] H. Herrmann and M. Beddig, Proc. Est. Acad. Sci. 67, 73 (2018).

[3] K. S. Thorne, Rev. Mod. Phys. 52, 299 (1980).

[4] E. Poisson and C. M. Will, Gravity: Newtonian, Post-Newtonian, Relativistic (Cambridge University Press, Cambridge, 2014).

[5] For closely related analyses, see A. J. Stone, Mol. Phys. 29, 1461 (1975); J. Phys. A 9, 485 (1976); A. Higuchi, J. Math. Phys. 28, 1553 (1987); T. Damour and B. R. Iyer, Phys. Rev. D 43, 3259 (1991); H. Ehrentraut and W. Muschik, ARI 51, 149 (1998).

[6] E. T. Newman and R. Penrose, J. Math. Phys. 7, 863 (1966).

[7] J. N. Goldberg, A. J. Macfarlane, E. T. Newman, F. Rohrlich, and E. C. G. Sudarshan, J. Math. Phys. 8, 2155 (1967).

[8] W. B. Campbell, J. Math. Phys. 12, 1763 (1971); G. F. T. del Castillo, 3-D Spinors, Spin-Weighted Functions, and Their Applications (Birkhäuser, Boston, 2003).
[9] V. A. Kostelecký and M. Mewes, Phys. Rev. D 80, 015020 (2009).

[10] V. A. Kostelecký and M. Mewes, Phys. Lett. B 766, 137 (2017).

[11] J. C. Long and V. A. Kostelecký, Phys. Rev. D 91, 092003 (2015); C. G. Shao, Y. J. Tan, W. H. Tan, S. Q. Yang, J. Luo, and M. E. Tobar, ibid. 91, 102007 (2015); C. G. Shao, Y. J. Tan, W. H. Tan, S. Q. Yang, J. Luo et al., Phys. Rev. Lett. 117, 071102 (2016); C. G. Shao, Y. F. Chen, Y. J. Tan, J. Luo, S. Q. Yang, and M. E. Tobar, Phys. Rev. D 94, 104061 (2016); Y. F. Chen, Y. J. Tan, and C. G. Shao, Symmetry 9, 219 (2017); C. G. Shao, Y. F. Chen, Y. J. Tan, S. Q. Yang, J. Luo, M. E. Tobar, J. C. Long, E. Weisman, and V. A. Kostelecký, Phys. Rev. Lett. 122, 011102 (2019).

[12] V. A. Kostelecký and S. Samuel, Phys. Rev. D 39, 683 (1989); V. A. Kostelecký and R. Potting, Nucl. Phys. B 359, 545 (1991).

[13] V. A. Kostelecký and R. Potting, Phys. Rev. D 51, 3923 (1995); D. Colladay and V. A. Kostelecký, ibid. 55, 6760 (1997); V. A. Kostelecký and R. Potting, ibid. 58, 116002 (1998); V. A. Kostelecký, ibid. 69, 105009 (2004). 
[14] A. Kostelecky and N. Russell, Rev. Mod. Phys. 83, 11 (2011).

[15] V. A. Kostelecký and M. Mewes, Phys. Lett. B 757, 510 (2016).

[16] V. A. Kostelecký and M. Mewes, Phys. Lett. B 779, 136 (2018); M. Mewes, Phys. Rev. D 99, 104062 (2019).
[17] M. Hamermesh, Group Theory and Its Application to Physical Problems (Addison Wesley, Reading, MA, 1962).

[18] H. Boerner, Representations of Groups (American Elsevier, New York, 1970).

[19] J. S. Frame, G. de B. Robinson, and R. M. Thrall, Can. J. Math. 6, 316 (1954). 